\title{
The multicomponent 2D Toda hierarchy: Discrete flows and string equations
}

\author{
Manuel Mañas, Luis Martínez Alonso, and Carlos Álvarez Fernández \\ Departamento de Física Teórica II, Universidad Complutense \\ 28040-Madrid, Spain \\ email: manuel.manas@fis.ucm.es
}

October 22, 2018

\begin{abstract}
The multicomponent 2D Toda hierarchy is analyzed through a factorization problem associated to an infinitedimensional group. A new set of discrete flows is considered and the corresponding Lax and Zakharov-Shabat equations are characterized. Reductions of block Toeplitz and Hankel bi-infinite matrix types are proposed and studied. Orlov-Schulman operators, string equations and additional symmetries (discrete and continuous) are considered. The continuous-discrete Lax equations are shown to be equivalent to a factorization problem as well as to a set of string equations. A congruence method to derive site independent equations is presented and used to derive equations in the discrete multicomponent $\mathrm{KP}$ sector (and also for its modification) of the theory as well as dispersive Whitham equations.
\end{abstract}

\section{Introduction}

This paper revisits the multicomponent 2D Toda hierarchy [30] from the point of view of the factorization problem associated to an infinite-dimensional group. Our main motivation is the recent discovery [3] of underlying integrable structures of multicomponent type in the theory of multiple orthogonal polynomials which is in turn connected to models of non-intersecting Brownian motions. Having in mind the fruitful applications of the Toda hierarchy to the theory of orthogonal polynomials and to the Hermitian random matrix model (see for instance [14- 21]), it is expected that the formalism of multicomponent integrable hierarchies can be similarly applied to the study and characterization of multiple orthogonal polynomials and non-intersecting Brownian motions. In particular, the semiclassical (dispersionless) limit of multicomponent integrable hierarchies should be relevant for the analysis of large $N$ ) type limits, see for instance 22. An important piece of the technique required for these applications was recently provided by Takasaki and Takebe [27, 28. Indeed, they proved that the universal Whitham hierarchy (genus 0 case) [16 can be obtained as a particular dispersionless limit of the multicomponent KP hierarchy.

The applications of the Toda hierarchy to the characterization of semiclassical limits make an essential use of the notion of string equations [14]-21]-[10. In recent years the formalism of string equations for dispersionless integrable hierarchies [26] has been much developed [32, 19] but, to our knowledge, a similar formalism for dispersive multicomponent integrable hierarchies is not yet available. One of the main goals of this paper is to extend the formalism of string equations to multicomponent 2D Toda hierarchies. In this sense the consideration of factorization problems for these hierarchies turns to be of great help in order to introduce basic ingredients such as discrete flows, Orlov-Schulman operators and additional symmetries.

The theory of the multicomponent KP hierarchy is discussed in length in the papers [15, 4, see also [20] for its applications to geometric nets of conjugate type. In [30] it was noticed that $\tau$ functions of a $2 N$-multicomponent KP provide solutions of the $N$-multicomponent Toda hierarchy. The introduction of integer parameters in the multicomponent KP hierarchy goes back to 8 and the corresponding discrete flows, which are used in two different ways in 15, 4, are essential for the derivation of the dispersionless Whitham hierarchy from the multicomponent KP hierarchy [27, 28, 229. In the present paper we introduce a set of discrete flows for the multicomponent 2D Toda hierarchy. Its role in the formulation of the corresponding dispersionless limits will be discussed in length in a forthcoming paper. 
The layout of the paper is as follows: In $\S 1$ we introduce a factorization problem in a Lie group as the one presented in [30. This factorization problem is rooted in the ideas used for the KP case in [24, 1], for the so called discrete KP hierarchy. Then we derive the continuous and discrete Lax equations for the multicomponent 2D Toda hierarchy. We notice that in our discussion the set of discrete flows, which to our knowledge where not considered before for this hierarchy, are formulated in equal footing to the continuous flows. We also show some examples of members of the hierarchy and, in particular, multicomponent equations of Toda type involving partial difference operators only, or combined partial difference and partial derivatives. We end this section with the formulation of several classes of reductions of the multicomponent $2 \mathrm{D}$ Toda hierarchy involving biinfinite block Toeplitz and Hankel matrices. The consideration of these types of reductions is motivated by their relevance in integrable hierarchies such as the infinite Toda hierarchy [2] or the Ablowitz-Ladik lattice hierarchy [6]. For some reductions we characterize solutions of the hierarchy which are periodic in the discrete variables. Moreover, for the Hankel case we get generalizations of the bigraded reduction, see [7, associated with extended flows of the 1-component 1D Toda hierarchy [12.

In $\S 2$ we formulate the theory of string equations for the multicomponent $2 \mathrm{D}$ Toda hierarchy. We start by defining the Orlov-Schulman operator 23 and then we derive its Lax equations from the factorization problem introduced in $\S 1$. We also show how the Lax equations imply in turn the factorization problem. In this way we stablish the equivalence between the factorization problem and the extended Lax formulation, involving discrete flows and the Orlov-Schulman operator, of the multicomponent 2D Toda hierarchy. Moreover, we also prove the equivalence between the extended Lax formulation and a particular type of string equations for the multicomponent 2D Toda hierarchy. This generalizes the result for the one-component case stablished in [26]. Finally, we use the factorization problem and the canonical pair of Lax and Orlov-Schulman operators to provide a natural formulation of the additional symmetries of the multicomponent $2 \mathrm{D}$ Toda hierarchy. As a consequence we characterize the string equations as invariance conditions under additional symmetries.

The paper ends with two appendices. In the first appendix the congruence method for deriving $n$-independent equations is shown. It is applied to get the main equations of the discrete multicomponent KP hierarchy: $N$-wave equations, Darboux equations and multiquadrilateral lattice equations [9]. We also use this method to formulate the dispersive Whitham equations in terms of scalar Lax and Orlov-Schulman opeartors, which constitute the starting point for the discussion of the dispersionless limits of the multicomponent 2D Toda hierarchy. Finally, the second appendix contains the proofs of the main Propositions of the paper.

\subsection{Lie algebra setting}

In this paper we only consider formal series expansions in the Lie group theoretic setup without any assumption on their convergency. We also remark that along the paper we use the following notations. For given Lie algebras $\mathfrak{g}_{1} \subset \mathfrak{g}_{2}$, and $X, Y \in \mathfrak{g}_{2}$ then $X=Y+\mathfrak{g}_{1}$ means $X-Y \in \mathfrak{g}_{1}$. For any Lie groups $G_{1} \subset G_{2}$ and $a, b \in G_{2}$ then $a=G_{2} \cdot b$ stands for $a \cdot b^{-1} \in G_{2}$. Let $\left\{E_{k l}\right\}_{k, l=1}^{N}$ be the standard basis $\left(E_{k l}\right)_{k^{\prime} l^{\prime}}=\delta_{l l^{\prime}} \delta_{k k^{\prime}}$ of $M_{N}(\mathbb{C})$ and $\rrbracket_{N}$ denote the identity matrix in $M_{N}(\mathbb{C})$. We also denote the algebra unit as 1 .

If $M_{N}(\mathbb{C})$ denotes the associative algebra of complex $N \times N$ matrices we will consider the linear space of sequences

$$
\begin{aligned}
f: \mathbb{Z} & \longrightarrow M_{N}(\mathbb{C}) \\
n & \longmapsto f(n) .
\end{aligned}
$$

The shift operator $\Lambda$ acts on these sequences as $(\Lambda f)(n):=f(n+1)$. A sequence $X: \mathbb{Z} \rightarrow M_{N}(\mathbb{C})$ acts by left multiplication in this space of sequences, and therefore we may consider expressions of the type $X \Lambda^{j}$, where $X=X(n)$ is a sequence which acts by left multiplication: $\left(X \Lambda^{j}\right)(f)(n):=X(n) \cdot f(n+j)$.

Moreover, defining the product $\left(X(n) \Lambda^{i}\right) \cdot\left(Y(n) \Lambda^{j}\right):=X(n) Y(n+i) \Lambda^{i+j}$ and extending it linearly we have that the set $\mathfrak{g}$ of Laurent series in $\Lambda$ is an associative algebra, which under the standard commutator is a Lie algebra. Observe that $\mathfrak{g}$ can be thought either as $M_{\mathbb{Z}}\left(M_{N}(\mathbb{C})\right.$ ), i.e. bi-infinite matrices with $M_{N}(\mathbb{C})$ coefficients, or as $M_{N}\left(M_{\mathbb{Z}}(\mathbb{C})\right)$, i.e. $N \times N$ matrices with coefficients bi-infinite matrices.

This Lie algebra has the following important splitting

$$
\mathfrak{g}=\mathfrak{g}_{+} \dot{+} \mathfrak{g}_{-},
$$

where

$$
\mathfrak{g}_{+}=\left\{\sum_{j \geqslant 0} X_{j}(n) \Lambda^{j}, \quad X_{j}(n) \in M_{N}(\mathbb{C})\right\}, \quad \mathfrak{g}_{-}=\left\{\sum_{j<0} X_{j}(n) \Lambda^{j}, \quad X_{j}(n) \in M_{N}(\mathbb{C})\right\},
$$


are Lie subalgebras of $\mathfrak{g}$ with trivial intersection.

\subsection{The Lie group and the factorization problem}

The group of linear invertible elements in $\mathfrak{g}$ will be denoted by $G$ and has $\mathfrak{g}$ as its Lie algebra, then the splitting (11) leads us to consider the following factorization of $g \in G$

$$
g=g_{-}^{-1} \cdot g_{+}, \quad g_{ \pm} \in G_{ \pm}
$$

where $G_{ \pm}$have $\mathfrak{g}_{ \pm}$as their Lie algebras. Explicitly, $G_{+}$is the set of invertible linear operators of the form $\sum_{j \geqslant 0} g_{j}(n) \Lambda^{j}$; while $G_{-}$is the set of invertible linear operators of the form $1+\sum_{j<0} g_{j}(n) \Lambda^{j}$.

An alternative factorization is the Gauss factorization

$$
g=\hat{g}_{-}^{-1} \cdot \hat{g}_{+}, \quad \hat{g}_{ \pm} \in \hat{G}_{ \pm}
$$

where $\hat{G}_{+}$is the set of invertible linear operators of the form $\hat{g}_{0,+}(n)+\sum_{j>0} \hat{g}_{j}(n) \Lambda^{j} ;$ with $\hat{g}_{0,+}: \mathbb{Z} \rightarrow \mathrm{GL}(N, \mathbb{C})$ an invertible upper triangular matrix, while $\hat{G}_{-}$is the set of invertible linear operators of the form $\hat{g}_{0,-}(n)^{-1}+$ $\sum_{j<0} \hat{g}_{j}(n) \Lambda^{j}$ with $\hat{g}_{0,-}: \mathbb{Z} \rightarrow \mathrm{GL}(N, \mathbb{C})$, such that $\hat{g}_{0,-}=\mathbb{\square}_{N}+A$, being $A$ a strictly lower triangular matrix in $M_{N}(\mathbb{C})$. If the factorization (3) exists then it will also exist (2) by defining $g_{+}=\hat{g}_{0,-} \cdot \hat{g}_{+}, \quad g_{-}=\hat{g}_{0,-} \cdot \hat{g}_{-} \cdot$ The elements $g$ with a factorization (3) are said to belong to the big cell [4], hence the factorization can be considered only locally. Thus, we will consider elements $g$ in the big cell so that the factorization (2) holds, avoiding the generation of additional problems connected with these local aspects.

Now we introduce two sets of indexes, $\mathbb{S}=\{1, \ldots, N\}$ and $\overline{\mathbb{S}}=\{\overline{1}, \ldots, \bar{N}\}$, of the same cardinality $N$. In what follows we will use letters $k, l$ and $\bar{k}, \bar{l}$ to denote elements in $\mathbb{S}$ and $\overline{\mathbb{S}}$, respectively. Furthermore, we will use letters $a, b, c$ to denote elements in $\mathcal{S}:=\mathbb{S} \cup \overline{\mathbb{S}}$.

We define the following operators $W_{0}, \bar{W}_{0} \in G$

$$
\begin{aligned}
& W_{0}:=\sum_{k=1}^{N} E_{k k} \Lambda^{s_{k}} \mathrm{e}^{\sum_{j=0}^{\infty} t_{j k} \Lambda^{j}} \\
& \bar{W}_{0}:=\sum_{k=1}^{N} E_{k k} \Lambda^{-s_{\bar{k}}} \mathrm{e}^{\sum_{j=1}^{\infty} t_{j \bar{k}} \Lambda^{-j}}
\end{aligned}
$$

where $s_{a} \in \mathbb{Z}, t_{j a} \in \mathbb{C}$ are deformation parameters, that in the sequel will play the role of discrete and continuous times, respectively.

The factorization problem Given an element $g \in G$, in the big cell, and a set of deformation parameters $\boldsymbol{s}=\left(s_{a}\right)_{a \in \mathcal{S}}, \boldsymbol{t}=\left(t_{j a}\right)_{a \in \mathcal{S}, j \in \mathbb{N}_{\mathrm{sg} a}}, \mathbb{N}_{+1}=\{0,1,2, \cdots\} \mathbb{N}_{-1}=\{1,2, \cdots\}$, we consider the factorization problem

$$
W_{0} \cdot g \cdot \bar{W}_{0}^{-1}=S(\boldsymbol{s}, \boldsymbol{t})^{-1} \cdot \bar{S}(\boldsymbol{s}, \boldsymbol{t}), \quad S \in G_{-} \text {and } \bar{S} \in G_{+},
$$

We will confine our analysis to the zero charge sector

$$
|s|:=\sum_{a \in \mathcal{S}} s_{a}=0,
$$

and consider small enough values of the continuous times. Observe that normally, but not always, the $t_{0 k}$ times are disregarded. The reason is the triviality of factorization associated with this deformations. In fact, if we have a solution of the factorization problem for $t_{0 k}=0$, with factors $S_{0}$ and $\bar{S}_{0}$, then the factors corresponding to the factorization with arbitrary $t_{0 k}$ are $S=\exp \left(\sum_{k=1}^{N} t_{0 k} E_{k k}\right) S_{0} \exp \left(-\sum_{k=1}^{N} t_{0 k} E_{k k}\right)$ and $\bar{S}=\exp \left(\sum_{k=1}^{N} t_{0 k} E_{k k}\right) \bar{S}_{0}$. The reason to consider them here is due to the reductions we will study later.

At this point we discuss some relevant subalgebras and subgroups which will play an important role hereafter. Firstly, we notice that an operator $A=\sum_{j \in \mathbb{Z}} A_{j}(n) \Lambda^{j}$ commutes with $\Lambda$ if and only if the coefficients $A_{j}$ do not

depend on $n$. Thus, the centralizer of $\Lambda$ is $\mathfrak{z} \Lambda:=\{A \in \mathfrak{g}:[A, \Lambda]=0\}=\left\{\sum_{j \in \mathbb{Z}} A_{j} \Lambda^{j}, A_{j} \in M_{N}(\mathbb{C})\right\}$. Observe that $\mathfrak{z} \Lambda \subset \mathfrak{g}$ is a Lie subalgebra as now $\Lambda$ commutes with the matrix coefficients of the Laurent expansions. Another interpretation is that we have block bi-infinite Toeplitz or Laurent operators $[5$.

A particular Abelian subalgebra $\mathfrak{h}$ of $\mathfrak{z} \Lambda$ is given by the centralizer of $\mathbb{C}\left\{\Lambda, E_{k k}\right\}_{k=1}^{N} ;$ i.e, $\mathfrak{h}:=\{A \in \mathfrak{g}:$ $\left.[A, \Lambda]=\left[A, E_{k k}\right]=0, k=1, \ldots, N\right\}=\left\{\sum_{j \in \mathbb{Z}} A_{j} \Lambda^{j}, A_{j} \in \operatorname{diag}(N, \mathbb{C})\right\}$ where $\operatorname{diag}(N, \mathbb{C})$ is the subalgebra of 
diagonal matrices of $M_{N}(\mathbb{C})$. Thus, $\mathfrak{h}$ is the set of Laurent series in $\Lambda$ with diagonal $n$-independent coefficients. There are two important subgroups: $G_{-} \cap \mathfrak{z}_{\Lambda}=\left\{1+c_{1} \Lambda^{-1}+c_{2} \Lambda^{-2}+\cdots, c_{j} \in M_{N}(\mathbb{C})\right\}$ and $G_{+} \cap \mathfrak{z}_{\Lambda}=$ $\left\{\bar{c}_{0}+\bar{c}_{1} \Lambda+\bar{c}_{2} \Lambda^{2}+\cdots, \quad \bar{C}_{0} \in \mathrm{GL}(N, \mathbb{C}), \bar{c}_{j} \in M_{N}(\mathbb{C}), j \geqslant 1\right\}$. Finally, we have the corresponding Abelian Lie subgroups $H:=G \cap \mathfrak{h}$ and $H_{ \pm}:=G_{ \pm} \cap \mathfrak{h}$ and $W_{0}, \bar{W}_{0}$ takes values in $H$.

We shall denote by $n \in \mathfrak{g}$ the multiplication operator by the sequence $\left\{n \rrbracket_{N}\right\}_{n \in \mathbb{Z}}$; i.e.

$$
n\{X(n)\}_{n \in \mathbb{Z}}=\{n X(n)\}_{n \in \mathbb{Z}} .
$$

Observe that $[\Lambda, n]=\Lambda$ and that for any $X \in \mathfrak{g}$ we have $X=\sum_{\substack{j \in \mathbb{Z} \\ i \geqslant 0}} X_{i j} n^{i} \Lambda^{j}, X_{i j} \in M_{N}(\mathbb{C})$. This expansion follows from the assumption that $X_{j}(n)=X_{0 j}+X_{i j} n+\cdots$. The set of operators commuting with $\Lambda, n$ and $E_{k k}, k=1, \ldots, N$ is given by $\left\{A \in \mathfrak{g}:[A, \Lambda]=[A, n]=\left[A, E_{k k}\right]=0, k=1, \ldots, N\right\}=\operatorname{diag}(N, \mathbb{C})$.

\section{Lax and Zakharov-Shabat equations}

\subsection{Dressing procedure. Lax and $C$ operators}

We now introduce important elements for the sequel of this paper

Definition 1. We define the dressing operators $W, \bar{W}$ as follows

$$
W:=S \cdot W_{0}, \quad \bar{W}:=\bar{S} \cdot \bar{W}_{0},
$$

In terms of these dressing operators the factorization problem([6) in $G$ reads

$$
W \cdot g=\bar{W}
$$

Observe that the expansions of the factors $S, \bar{S}$

$$
\begin{aligned}
& S=\mathbb{1}_{N}+\varphi_{1}(n) \Lambda^{-1}+\varphi_{2}(n) \Lambda^{-2}+\cdots \in G_{-}, \\
& \bar{S}=\bar{\varphi}_{0}(n)+\bar{\varphi}_{1}(n) \Lambda+\bar{\varphi}_{2}(n) \Lambda^{2}+\cdots \in G_{+} .
\end{aligned}
$$

Sometimes we will use the notation

$$
\beta:=\varphi_{1}, \quad \mathrm{e}^{\phi}:=\bar{\varphi}_{0} .
$$

We have the following expressions

$$
\begin{aligned}
& W=\left(\mathbb{\square}_{N}+\varphi_{1}(n) \Lambda^{-1}+\varphi_{2}(n) \Lambda^{-2}+\cdots\right) \cdot\left(\sum_{k=1}^{N} E_{k k} \Lambda^{s_{k}} \exp \left(\sum_{j=0}^{\infty} t_{j k} \Lambda^{j}\right)\right) \\
& \bar{W}=\left(\bar{\varphi}_{0}(n)+\bar{\varphi}_{1}(n) \Lambda+\bar{\varphi}_{2}(n) \Lambda^{2}+\cdots\right) \cdot\left(\sum_{k=1}^{N} E_{k k} \Lambda^{-s_{\bar{k}}} \exp \left(\sum_{j=1}^{\infty} t_{j \bar{k}} \Lambda^{-j}\right)\right)
\end{aligned}
$$

Other important objects are

Definition 2. The Lax operators $L, \bar{L}, C_{k l}, \bar{C}_{k l}, \mathcal{C}_{k l}, \overline{\mathcal{C}}_{k l} \in \mathfrak{g}$ are given by

$$
\begin{aligned}
L & :=W \cdot \Lambda \cdot W^{-1}, & \bar{L} & :=\bar{W} \cdot \Lambda \cdot \bar{W}^{-1}, \\
C_{k l} & :=W \cdot E_{k l} \cdot W^{-1}, & \bar{C}_{k l} & :=\bar{W} \cdot E_{k l} \cdot \bar{W}^{-1} \\
\mathcal{C}_{k l} & :=S \cdot E_{k l} \cdot S^{-1}, & \overline{\mathcal{C}}_{k l} & :=\bar{S} \cdot E_{k l} \cdot \bar{S}^{-1} .
\end{aligned}
$$

Notice that in the above definitions of $L, \bar{L}, C_{k k}$ and $\bar{C}_{k k}$-as $W_{0}, \bar{W}_{0} \in H$ - we may replace the dressing operators $W$ and $\bar{W}$ by $S$ and $\bar{S}$, respectively. A straightforward calculations yields

Proposition 1. 1. The following relations holds

$$
\begin{aligned}
& \left.C_{k l}=L^{s_{k}-s_{l}} \exp \left(\sum_{j=0}^{\infty}\left(t_{j k}-t_{j l}\right) L^{j}\right)\right) \mathcal{C}_{k l}, \\
& \left.\bar{C}_{k l}=\bar{L}^{-s_{\bar{k}}+s_{\bar{l}}} \exp \left(\sum_{j=1}^{\infty}\left(t_{j \bar{k}}-t_{j \bar{l}}\right) \bar{L}^{-j}\right)\right) \overline{\mathcal{C}}_{k l} .
\end{aligned}
$$


2. The Lax operators have the following expansions

$$
\begin{aligned}
L & =\Lambda+u_{1}(n)+u_{2}(n) \Lambda^{-1}+\cdots, & \bar{L}^{-1} & =\bar{u}_{0}(n) \Lambda^{-1}+\bar{u}_{1}(n)+\bar{u}_{2}(n) \Lambda+\cdots, \\
\mathcal{C}_{k l} & =E_{k l}+C_{k l, 1}(n) \Lambda^{-1}+C_{k l, 2}(n) \Lambda^{-2}+\cdots, & \overline{\mathcal{C}}_{k l} & =\bar{C}_{k l, 0}(n)+\bar{C}_{k l, 1}(n) \Lambda+\bar{C}_{k l, 2}(n) \Lambda^{2}+\cdots .
\end{aligned}
$$

3. These operators fulfill

$$
\begin{gathered}
\mathbb{\square}_{N}=\sum_{k=1}^{N} C_{k k}, \quad \square_{N}=\sum_{k=1}^{N} \bar{C}_{k k}, \\
C_{k l} C_{k^{\prime} l^{\prime}}=\delta_{l k^{\prime}} C_{k l^{\prime}}, \quad C_{k l} L=L C_{k l}, \\
\bar{C}_{k l} \bar{C}_{k^{\prime} l^{\prime}}=\delta_{l k^{\prime}} \bar{C}_{k l^{\prime}}, \quad \bar{C}_{k l} \bar{L}=\bar{L} \bar{C}_{k l},
\end{gathered}
$$

\subsection{Lax and Zakharov-Shabat equations}

In this section we will use the factorization problem (9) to derive two sets of equations: Lax equations and Zakharov-Shabat equations, and we will show they all are equivalent. Let us first introduce some convenient notation

Definition 3. 1.

$$
\partial_{j a}:=\frac{\partial}{\partial t_{j a}},
$$

2. The zero-charge shifts $T_{K}$ for $K=(a, b)$ are defined as follows

$$
s_{a} \rightarrow s_{a}+1, \quad s_{b} \rightarrow s_{b}-1,
$$

and all the others discrete variables remain unchanged.

3.

$$
\begin{aligned}
\theta_{j a}:=\partial_{j a} W_{0} \cdot W_{0}^{-1}, & \bar{\theta}_{j a}:=\bar{\partial}_{j a} \bar{W}_{0} \cdot \bar{W}_{0}^{-1}, \\
q_{K}:=T_{K} W_{0} \cdot W_{0}^{-1}, & \bar{q}_{K}:=T_{K} \bar{W}_{0} \cdot \bar{W}_{0}^{-1}, \quad K=(a, b) .
\end{aligned}
$$

4.

$$
\begin{array}{ll}
C_{a a}:=W \pi_{a} W^{-1} & \bar{C}_{a a}:=\bar{W} \bar{\pi}_{a} \bar{W}^{-1}, \\
\mathcal{R}_{j a}:=W \theta_{j a} W^{-1}, & \overline{\mathcal{R}}_{j a}:=\bar{W} \bar{\theta}_{a j} \bar{W}^{-1}, \quad \mathcal{U}_{K}:=W q_{K} W^{-1}, \quad \overline{\mathcal{U}}_{K}:=\bar{W} \bar{q}_{K} \bar{W}^{-1} .
\end{array}
$$

5 .

$$
\begin{array}{rlr}
B_{j a} & :=\mathcal{R}_{j a}-\left(\mathcal{R}_{j a}-\overline{\mathcal{R}}_{j a}\right)_{-}=\overline{\mathcal{R}}_{j a}+\left(\mathcal{R}_{j a}-\overline{\mathcal{R}}_{j a}\right)_{+} \in \mathfrak{g}, & \left(\mathcal{R}_{j a}-\overline{\mathcal{R}}_{j a}\right)_{ \pm} \in \mathfrak{g}_{ \pm} \\
\omega_{K}:=\left(\mathcal{U}_{k} \cdot \overline{\mathcal{U}}_{K}^{-1}\right)_{-} \cdot \mathcal{U}_{K}=\left(\mathcal{U}_{k} \cdot \overline{\mathcal{U}}_{K}^{-1}\right)_{+} \cdot \overline{\mathcal{U}}_{K} \in G, & \left(\mathcal{U}_{K} \overline{\mathcal{U}}_{K}^{-1}\right)_{ \pm} \in G_{ \pm}
\end{array}
$$

Notice that if

$$
\pi_{a}:=\left\{\begin{array}{ll}
E_{k k}, & a=k \in \mathbb{S}, \\
0, & a \in \overline{\mathbb{S}},
\end{array} \quad \bar{\pi}_{a}:= \begin{cases}0, & a \in \mathbb{S}, \\
E_{k k}, & a \in \overline{\mathbb{S}} \text { and } a=\bar{k} \text { for some } k \in \mathbb{S} .\end{cases}\right.
$$

we can write

$$
\begin{array}{rlrl}
\theta_{j a} & =\pi_{a} \Lambda^{j}, & \bar{\theta}_{j a} & =\bar{\pi}_{a} \Lambda^{-j}, \\
q_{K} & =\rrbracket_{N}+\pi_{a}\left(\Lambda-\rrbracket_{N}\right)+\pi_{b}\left(\Lambda^{-1}-\rrbracket_{N}\right), & & \bar{q}_{K} \\
=\rrbracket_{N}+\bar{\pi}_{a}\left(\Lambda^{-1}-\rrbracket_{N}\right)+\bar{\pi}_{b}\left(\Lambda-\rrbracket_{N}\right), \quad K=(a, b) .
\end{array}
$$

Observe that all the shift operators preserve the zero charge sector and form a commutative group

$$
\begin{aligned}
& T_{K} T_{K^{\prime}}=T_{K^{\prime}} T_{K}, \\
& T_{(a, b)} T_{(b, a)}=\mathrm{id},
\end{aligned}
$$

satisfying the following cohomological relations

$$
T_{(a, b)} T_{(b, c)} T_{(c, a)}=\mathrm{id} .
$$


Proposition 2. The relations (20)-(22) are equivalent to

$$
T_{(a, b)} T_{(b, c)}=T_{(b, c)} T_{(a, b)}=T_{(a, c)}
$$

where $T_{(a, a)}=\mathrm{id}$.

Proof. See Appendix B.

Also notice that $B_{j k}=\left(C_{k k} L^{j}\right)_{+}, B_{j \bar{k}}=\left(\bar{C}_{k k} \bar{L}^{-j}\right)_{-}$and that (18) and (19) gives

$$
\omega_{K}=\pi_{a} \Lambda+a_{K}+\bar{a}_{K} \Lambda^{-1},
$$

for some matrix sequences $a_{K}(n)$ and $\bar{a}_{K}(n)$.

The factorization problem (6) implies that the partial differential equations

$$
\partial_{j a} W \cdot W^{-1}=\partial_{j a} S \cdot S^{-1}+S \cdot \theta_{j a} \cdot S^{-1}=\partial_{j a} \bar{S} \cdot \bar{S}^{-1}+\bar{S} \cdot \bar{\theta}_{j a} \cdot \bar{S}^{-1}=\partial_{j a} \bar{W} \cdot \bar{W}^{-1},
$$

and partial difference equations

$$
T_{K} W \cdot W^{-1}=T_{K} S \cdot q_{K} \cdot S^{-1}=T_{K} \bar{S} \cdot \bar{q}_{K} \cdot \bar{S}^{-1}=T_{K} \bar{W} \cdot \bar{W}^{-1}
$$

hold.

From the previous proposition we derive the following linear systems for the dressing operators and Lax equations for the Lax operators, and its compatibility conditions

Theorem 1. 1. The dressing operators are subject to

$$
\begin{array}{ll}
\partial_{j a} W=B_{j a} \cdot W, & \partial_{j a} \bar{W}=B_{j a} \cdot \bar{W}, \\
T_{K} W=\omega_{K} \cdot W, & T_{K} \bar{W}=\omega_{K} \cdot \bar{W} .
\end{array}
$$

2. The Lax equations

$$
\begin{array}{llll}
\partial_{j a} L=\left[B_{j a}, L\right], & \partial_{j a} \bar{L}=\left[B_{j a}, \bar{L}\right], & \partial_{j a} C_{k k}=\left[B_{j a}, C_{k k}\right], & \partial_{j a} \bar{C}_{k k}=\left[B_{j a}, \bar{C}_{k k}\right], \\
T_{K} L=\omega_{K} \cdot L \cdot \omega_{K}^{-1}, & T_{K} \bar{L}=\omega_{K} \cdot \bar{L} \cdot \omega_{K}^{-1}, & T_{K} C_{k k}=\omega_{K} \cdot C_{k k} \cdot \omega_{K}^{-1}, & T_{K} \bar{C}_{k k}=\omega_{K} \cdot \bar{C}_{k k} \cdot \omega_{K}^{-1}
\end{array}
$$

are satisfied.

3. The following Zakharov-Shabat equations hold

$$
\begin{gathered}
\partial_{j a} B_{i b}-\partial_{i b} B_{j a}+\left[B_{i b}, B_{j a}\right]=0, \\
T_{K} B_{j a}=\partial_{j a} \omega_{K} \cdot \omega_{K}^{-1}+\omega_{K} \cdot B_{j a} \cdot \omega_{K}^{-1}, \\
T_{K} \omega_{K^{\prime}} \cdot \omega_{K}=T_{K^{\prime}} \omega_{K} \cdot \omega_{K^{\prime}} .
\end{gathered}
$$

Proof. 1. First, observe that (25) implies $\partial_{j a} S \cdot S^{-1}+\mathcal{R}_{j a}=\partial_{j a} \bar{S} \cdot \bar{S}^{-1}+\overline{\mathcal{R}}_{j a}$, and therefore $\partial_{j a} S \cdot S^{-1}=$ $-\left(\mathcal{R}_{j a}-\overline{\mathcal{R}}_{j a}\right)_{-} \in \mathfrak{g}_{-}$and $\partial_{j a} \bar{S} \cdot \bar{S}^{-1}=\left(\mathcal{R}_{j a}-\overline{\mathcal{R}}_{j a}\right)_{+} \in \mathfrak{g}_{+}$so that using again (25) we get

$$
\partial_{j a} W \cdot W^{-1}=-\left(\mathcal{R}_{j a}-\overline{\mathcal{R}}_{j a}\right)_{-}+\mathcal{R}_{j a}=B_{j a}=\left(\mathcal{R}_{j a}-\overline{\mathcal{R}}_{j a}\right)_{+}+\overline{\mathcal{R}}_{j a}=\partial_{j a} \bar{W} \cdot \bar{W}^{-1}
$$

Equation (26) implies

$$
T_{K} W \cdot W^{-1}=T_{K} S \cdot q_{K} \cdot S^{-1}=T_{K} S \cdot S^{-1} \cdot \mathcal{U}_{K}=T_{K} \bar{S} \cdot \bar{S}^{-1} \cdot \overline{\mathcal{U}}_{K}=T_{K} \bar{S} \cdot \bar{q}_{K} \cdot \bar{S}^{-1}=T_{K} \bar{W} \cdot \bar{W}^{-1}
$$

so that $\left(T_{K} S \cdot S^{-1}\right)^{-1} \cdot\left(T_{K} \bar{S} \cdot \bar{S}^{-1}\right)=\mathcal{U}_{K} \cdot \overline{\mathcal{U}}_{K}^{-1}$ and we conclude $T_{K} S \cdot S^{-1}=\left(\mathcal{U}_{K} \cdot \overline{\mathcal{U}}_{K}^{-1}\right)_{-} \in G_{-}$and $T_{K} \bar{S} \cdot \bar{S}^{-1}=\left(\mathcal{U}_{K} \cdot \overline{\mathcal{U}}_{K}^{-1}\right)_{+} \in G_{+}$which introduced back in (35) gives

$$
\begin{aligned}
T_{K} W \cdot W^{-1}=T_{K} S \cdot q_{K} \cdot S^{-1}=\left(\mathcal{U}_{K} \cdot \overline{\mathcal{U}}_{K}^{-1}\right)_{-} & \cdot \mathcal{U}_{K}=\omega_{K} \\
& =\left(\mathcal{U}_{K} \cdot \overline{\mathcal{U}}_{K}^{-1}\right)_{+} \cdot \overline{\mathcal{U}}_{K}=T_{K} \bar{S} \cdot \bar{q}_{K} \cdot \bar{S}^{-1}=T_{K} \bar{W} \cdot \bar{W}^{-1} .
\end{aligned}
$$


2. From the definition (12) we get

$$
\begin{aligned}
\partial_{j a} L & =\left[\partial_{j a} W \cdot W^{-1}, L\right], & \partial_{j a} \bar{L} & =\left[\partial_{j a} \bar{W} \cdot \bar{W}^{-1}, \bar{L}\right], \\
\partial_{j a} C_{k k} & =\left[\partial_{j a} \bar{W} \cdot \bar{W}^{-1}, C_{k k}\right], & \partial_{j a} \bar{C}_{k k} & =\left[\partial_{j a} \bar{W} \cdot \bar{W}^{-1}, \bar{C}_{k k}\right], \\
T_{K} L & =\left(T_{K} W \cdot W^{-1}\right) \cdot L \cdot\left(T_{K} W \cdot W^{-1}\right)^{-1}, & T_{K} \bar{L} & =\left(T_{K} \bar{W} \cdot \bar{W}^{-1}\right) \cdot \bar{L} \cdot\left(T_{K} \bar{W} \cdot \bar{W}^{-1}\right)^{-1}, \\
T_{K} C_{k k} & =\left(T_{K} W \cdot W^{-1}\right) \cdot C_{k k} \cdot\left(T_{K} W \cdot W^{-1}\right)^{-1}, & T_{K} \bar{C}_{k k} & =\left(T_{K} \bar{W} \cdot \bar{W}^{-1}\right) \cdot \bar{C}_{k k} \cdot\left(T_{K} \bar{W} \cdot \bar{W}^{-1}\right)^{-1} .
\end{aligned}
$$

and using (27) and (28) we find (29) and (30), respectively.

3. The compatibility of (27) and (28) imply (31)-(33)

Observe also that the trivial flows $t_{0 k}, k=1, \ldots, N$ are immediately integrated and if $L_{0}, \bar{L}_{0}, C_{k l}$ and $\bar{C}_{k l}$ are the Lax and $C$ operators corresponding to $t_{0 k}=0$ for arbitrary $t_{k 0}$ we only need to conjugate these operators with $\exp \left(\sum_{k=1}^{N} E_{k k} t_{k 0}\right)$.

The compatibility conditions (31)-(33) for operators $B_{j a}$ and $\omega_{K}$ formally imply the local existence of a matrix potential $\xi$ such that $B_{j a}=\partial_{j a} \xi \cdot \xi^{-1}$ and $\omega_{K}=T_{K} \xi \cdot \xi^{-1}$; here, the potential $\xi$ is a map to the Lie group $G$ depending on the variables $\left\{t_{j a}, s_{a}\right\}$ i. Moreover, any operator $\xi$ generates a gauge transformation so that $B_{j a} \rightarrow \partial_{j a} \xi \cdot \xi^{-1}+\xi \cdot B_{j a} \cdot \xi^{-1}$ and $\omega_{K} \rightarrow T_{K} \xi \cdot \omega_{K} \cdot \xi^{-1}$, providing new solutions of (31)-(33).

Proposition 3. The relations

$$
\left(T_{(a, b)} \omega_{(b, c)}\right) \omega_{(a, b)}=\left(T_{(b, c)} \omega_{(a, b)}\right) \omega_{(b, c)}=\omega_{(a, c)} .
$$

and the compatibility conditions (33) are equivalent.

Proof. See Appendix B.

We have seen that the Lax equations (29)-(30) and Zakharov-Shabat equations (31)-(33) appear as consequence of the factorization problem. The compatibility conditions for the Lax equations are satisfied if the Zakharov-Shabat equations hold. It is a standard fact in the theory of Integrable Systems that by construction the Lax equations imply the Zakharov-Shabat equations and therefore the system is compatible. In [30] is proven this fact for the differential equations (not the difference nor difference-differential equations) involved in the multicomponent 2D Toda hierarchy, that is that (29) $\Rightarrow(31)$. Here we give an extended proof in order to include the continuous-discrete and discrete-discrete cases.

Proposition 4. Let $\left\{L, C_{k k}\right\}_{k=1}^{N} \subset \mathfrak{g}$ and $\left\{\bar{L}, \bar{C}_{k k}\right\}_{k=1}^{N} \subset \mathfrak{g}$ be two sets, composed each of them of commuting operators, consider functions $\mathcal{R}_{j a} \in \mathfrak{g}, \mathcal{U}_{K} \in G$ of $L, C_{11}, \ldots, C_{k k}$ and $\overline{\mathcal{R}}_{j a} \in \mathfrak{g}, \overline{\mathcal{U}}_{K} \in G$ of $\bar{L}, \bar{C}_{11}, \ldots, \bar{C}_{k k}$, and define $B_{j a}$ and $\omega_{K}$ according to (19), then the Lax equations (29) and (30) imply the Zakharov-Shabat equations (31) - (33).

Proof. See Appendix B.

\subsection{The multicomponent Toda equations}

Here we write down some of the nonlinear partial differential-difference equations appearing as a consequence of the factorization problem (99). From (34) and (36), taking into account that $S \in G_{-}$and $\bar{S} \in G_{+}$, we deduce the following

Corollary 1. We have the expressions

$$
\begin{aligned}
B_{1 a} & =\pi_{a} \Lambda+U_{a}+\bar{U}_{a} \Lambda^{-1}, \\
\omega_{K} & :=\pi_{a} \Lambda+a_{K}+\bar{a}_{K} \Lambda^{-1}, \quad K=(a, b),
\end{aligned}
$$


where the coefficients have the alternative expressions

$$
\begin{aligned}
& U_{a}:=\beta(n) \pi_{a}-\pi_{a} \beta(n+1)= \begin{cases}\partial_{1 a}\left(\mathrm{e}^{\phi(n)}\right) \cdot \mathrm{e}^{-\phi(n)}, & a \in \mathbb{S} \\
0, & a \in \overline{\mathbb{S}}\end{cases} \\
& \bar{U}_{a}=\mathrm{e}^{\phi(n)} \bar{\pi}_{a} \mathrm{e}^{-\phi(n-1)}= \begin{cases}0, & a \in \mathbb{S}, \\
\partial_{1 a} \beta(n), & a \in \overline{\mathbb{S}},\end{cases} \\
& a_{K}:=\mathbb{\square}_{N}-\pi_{a}-\pi_{b}+T_{K} \beta(n) \pi_{a}-\pi_{a} \beta(n+1)= \begin{cases}\mathrm{e}^{T_{K} \phi(n)} \cdot\left(\mathbb{\square}_{N}-\bar{\pi}_{b}\right) \cdot \mathrm{e}^{-\phi(n)}, & a \in \mathbb{S}, \\
\mathbb{V}_{N}-\pi_{b}, & a \in \mathbb{S},\end{cases} \\
& \bar{a}_{K}:=\mathrm{e}^{T_{K} \phi(n)} \bar{\pi}_{a} \mathrm{e}^{-\phi(n-1)}= \begin{cases}0, & a \in \mathbb{S}, \\
T_{K} \beta(n)\left(\mathbb{\square}_{N}-\pi_{b}\right)-\left(\mathbb{\square}_{N}-\pi_{b}\right) \beta(n)+\pi_{b}, & a \in \overline{\mathbb{S}} .\end{cases}
\end{aligned}
$$

From (39) we deduce the following set of nonlinear partial differential-difference equations

$$
\left\{\begin{aligned}
\beta(n) E_{k k}-E_{k k} \beta(n+1) & =\partial_{1 k}\left(\mathrm{e}^{\phi(n)}\right) \cdot \mathrm{e}^{-\phi(n)}, \\
\partial_{1 \bar{k}} \beta(n) & =\mathrm{e}^{\phi(n)} E_{k k} \mathrm{e}^{-\phi(n-1)}, \\
T_{(k, b)} \beta(n) E_{k k}-E_{k k} \beta(n+1)+\rrbracket_{N}-E_{k k}-\pi_{b} & =\mathrm{e}^{T_{(k, b)} \phi(n)} \cdot\left(\mathbb{\square}_{N}-\bar{\pi}_{b}\right) \cdot \mathrm{e}^{-\phi(n)}, \\
T_{(\bar{k}, b)} \beta(n)\left(\rrbracket_{N}-\pi_{b}\right)-\left(\mathbb{\square}_{N}-\pi_{b}\right) \beta(n)+\pi_{b} & =\mathrm{e}^{T_{(\bar{k}, b)} \phi(n)} \cdot E_{k k} \cdot \mathrm{e}^{-\phi(n-1)} .
\end{aligned}\right.
$$

These equations constitute what we call the multicomponent Toda equations. Observe that if we cross the two first equations we get

$$
\partial_{1 \bar{k}^{\prime}}\left(\partial_{1 k}\left(\mathrm{e}^{\phi(n)}\right) \cdot \mathrm{e}^{-\phi(n)}\right)=\mathrm{e}^{\phi(n)} E_{k^{\prime} k^{\prime}} \mathrm{e}^{-\phi(n-1)} E_{k k}-E_{k k} \mathrm{e}^{\phi(n+1)} E_{k^{\prime} k^{\prime}} \mathrm{e}^{-\phi(n)}
$$

which is the matrix extension of the $2 \mathrm{D}$ Toda equation, which appears for $N=1$ :

$$
\partial_{1} \partial_{\overline{1}}(\phi(n))=\mathrm{e}^{\phi(n)-\phi(n-1)}-\mathrm{e}^{\phi(n+1)-\phi(n)} .
$$

If in the last equation we set $b=\bar{l} \in \overline{\mathcal{S}}$ we have

$$
\Delta_{(\bar{k}, \bar{l})} \beta(n)=\mathrm{e}^{T_{(\bar{k}, \bar{l}} \phi(n)} \cdot E_{k k} \cdot \mathrm{e}^{-\phi(n-1)} .
$$

which when considered simultaneously with the first gives

$$
\Delta_{\left(\bar{k}^{\prime}, \bar{l}\right)}\left(\partial_{1 k}\left(\mathrm{e}^{\phi(n)}\right) \cdot \mathrm{e}^{-\phi(n)}\right)=\mathrm{e}^{T_{\left(\bar{k}^{\prime}, \bar{l}\right)} \phi(n)} \cdot E_{k^{\prime} k^{\prime}} \cdot \mathrm{e}^{-\phi(n-1)} E_{k k}-E_{k k} \mathrm{e}^{T_{\left(\bar{k}^{\prime}, \bar{l}\right.} \phi(n+1)} \cdot E_{k^{\prime} k^{\prime}} \cdot \mathrm{e}^{-\phi(n)}
$$

which is a Toda type equation. A completely discrete equation appears, for example, when crossing the two last equations, i.e.

$$
\Delta_{\left(\bar{k}^{\prime}, \bar{l}\right)}\left(\mathrm{e}^{T_{(k, b)} \phi(n)} \cdot\left(\mathbb{\square}_{N}-\bar{\pi}_{b}\right) \cdot \mathrm{e}^{-\phi(n)}\right)=T_{(k, b)}\left(\mathrm{e}^{T_{\left(\bar{k}^{\prime}, \bar{l}\right)} \phi(n)} \cdot E_{k^{\prime} k^{\prime}} \cdot \mathrm{e}^{-\phi(n-1)}\right) E_{k k}-E_{k k} \mathrm{e}^{T_{\left.\bar{k}^{\prime}, \bar{l}\right)} \phi(n+1)} \cdot E_{k^{\prime} k^{\prime}} \cdot \mathrm{e}^{-\phi(n)} .
$$

So forth and so on we may get a set of continuous-discrete set of Toda type equations. Finally, observe that when $N=1$ we only have the shift $T_{\left(s_{1}, s_{\overline{1}}\right)}$ which corresponds to a shift $n \rightarrow n+1$.

\subsection{Block Toeplitz/Hankel reductions}

We now consider some reductions of the multicomponent 2D Toda hierarchy. In the first place we discuss an extension of the periodic reduction [30] and the bigraded reduction [7] to the multicomponent case, which we call Toeplitz/Hankel reduction. Finally we discuss an extension of the 1 dimensional reduction discussed in 30. These reductions are relevant when we work with semi-infinite cases, as in the construction of families of bi-orthogonal and orthogonal matrix polynomials, to be published elsewhere.

Given a set $\left\{\ell_{a}\right\}_{a \in \mathcal{S}} \subset \mathbb{Z}$ we seek for initial conditions $g$ satisfying

$$
g \cdot\left(\sum_{k=1}^{N} E_{k k} \Lambda^{-\ell_{\bar{k}}}\right)=\left(\sum_{k=1}^{N} E_{k k} \Lambda^{\ell_{k}}\right) \cdot g .
$$


The relation (41) gives the following constraints over the Lax operators

$$
\sum_{k=1}^{N} C_{k k} L^{\ell_{k} j}=\sum_{k=1}^{N} \bar{C}_{k k} \bar{L}^{-\ell_{\bar{k}} j}
$$

for any $j \in \mathbb{Z}$. To proceed further in the analysis of these reductions we define the sets

$$
\mathbb{S}_{ \pm}:=\left\{a \in \mathbb{S}: \pm \ell_{a}>0\right\}, \quad \mathbb{S}_{0}:=\left\{a \in \mathbb{S}: \ell_{a}=0\right\}, \quad \overline{\mathbb{S}}_{ \pm}:=\left\{a \in \overline{\mathbb{S}}: \pm \ell_{a}>0\right\}, \quad \overline{\mathbb{S}}_{0}:=\left\{a \in \overline{\mathbb{S}}: \ell_{a}=0\right\},
$$

so that $\mathbb{S}=\mathbb{S}_{+} \cup \mathbb{S}_{0} \cup \mathbb{S}_{-}$, and $\overline{\mathbb{S}}=\overline{\mathbb{S}}_{+} \cup \overline{\mathbb{S}}_{0} \cup \overline{\mathbb{S}}_{-}$.

Proposition 5. If (42) holds then we have

1. The dressing operators are subject to

$$
\begin{gathered}
\left(\sum_{a \in \mathbb{S}_{+} \cup \mathbb{S}_{0} \cup \bar{S}_{+}} \partial_{j \ell_{a}, a}\right)(W)=W \sum_{k=1}^{N} E_{k k} \Lambda^{j \ell_{k}}, \quad\left(\sum_{a \in \mathbb{S}_{+} \cup \mathbb{S}_{0} \cup \bar{S}_{+}} \partial_{j \ell_{a}, a}\right)(\bar{W})=\bar{W} \sum_{k=1}^{N} E_{k k} \Lambda^{-j \ell_{\bar{k}}}, \\
\left(\sum_{a \in \mathbb{S}_{-} \cup \mathbb{S}_{0} \cup \overline{\mathbb{S}}_{-}} \partial_{j\left|\ell_{a}\right|, a}\right)(W)=W \sum_{k=1}^{N} E_{k k} \Lambda^{-j \ell_{k}}, \quad\left(\sum_{a \in \mathbb{S}_{-} \cup \mathbb{S}_{0} \cup \overline{\mathbb{S}}_{-}} \partial_{j\left|\ell_{a}\right|, a}\right)(\bar{W})=\bar{W} \sum_{k=1}^{N} E_{k k} \Lambda^{j \ell_{\bar{k}}},
\end{gathered}
$$

for $j>0$.

2. The Lax operators are invariant:

$$
\begin{aligned}
& \left(\sum_{a \in \mathbb{S}_{+} \cup \mathbb{S}_{0} \cup \bar{S}_{+}} \partial_{j \ell_{a}, a}\right)(L)=\left(\sum_{a \in \mathbb{S}_{+} \cup \mathbb{S}_{0} \cup \bar{S}_{+}} \partial_{j \ell_{a}, a}\right)(\bar{L})=0, \\
& \left(\sum_{a \in \mathbb{S}_{-} \cup \mathbb{S}_{0} \cup \bar{S}_{-}} \partial_{j\left|\ell_{a}\right|, a}\right)(L)=\left(\sum_{a \in \mathbb{S}_{-} \cup \mathbb{S}_{0} \cup \bar{S}_{-}} \partial_{j\left|\ell_{a}\right|, a}\right)(\bar{L})=0,
\end{aligned}
$$

where $j>0$.

Moreover, if

$$
\sum_{a \in \mathcal{S}} \ell_{a}=0
$$

then,

1. The dressing operators fulfill

$$
\begin{aligned}
& W\left(s_{1}+\ell_{1}, \ldots, s_{N}+\ell_{N}, s_{\overline{1}}+\ell_{\overline{1}}, \ldots, s_{\bar{N}}+\ell_{\bar{N}}\right)=W\left(s_{1}, \ldots, s_{N}, s_{\overline{1}}, \ldots, s_{\bar{N}}\right) \sum_{k=1}^{N} E_{k k} \Lambda^{\ell_{k}} \\
& \bar{W}\left(s_{1}+\ell_{1}, \ldots, s_{N}+\ell_{N}, s_{\overline{1}}+\ell_{\overline{1}}, \ldots, s_{\bar{N}}+\ell_{\bar{N}}\right)=\bar{W}\left(s_{1}, \ldots, s_{N}, s_{\overline{1}}, \ldots, s_{\bar{N}}\right) \sum_{k=1}^{N} E_{k k} \Lambda^{-\ell_{\bar{k}}}
\end{aligned}
$$

2. The Lax operators are periodic

$$
\begin{aligned}
L\left(s_{1}+\ell_{1}, \ldots, s_{N}+\ell_{N}, s_{\overline{1}}+\ell_{\overline{1}}, \ldots, s_{\bar{N}}+\ell_{\bar{N}}\right) & =L\left(s_{1}, \ldots, s_{N}, s_{\overline{1}}, \ldots, s_{\bar{N}}\right) \\
\bar{L}\left(s_{1}+\ell_{1}, \ldots, s_{N}+\ell_{N}, s_{\overline{1}}+\ell_{\overline{1}}, \ldots, s_{\bar{N}}+\ell_{\bar{N}}\right) & =\bar{L}\left(s_{1}, \ldots, s_{N}, s_{\overline{1}}, \ldots, s_{\bar{N}}\right) .
\end{aligned}
$$

To prove this we need the

Lemma 1. If (42) holds then for $j>0$ we have

$$
\begin{aligned}
\sum_{a \in \mathbb{S}_{+} \cup \mathbb{S}_{0} \cup \overline{\mathbb{S}}_{+}} B_{j \ell_{a}, a} & =\sum_{k=1}^{N} C_{k k} L^{\ell_{k} j}=\sum_{k=1}^{N} \bar{C}_{k k} \bar{L}^{-\ell_{\bar{k}} j}, \\
\sum_{a \in \mathbb{S}_{-} \cup \mathbb{S}_{0} \cup \bar{S}_{-}} B_{j\left|\ell_{a}\right|, a} & =\sum_{k=1}^{N} C_{k k} L^{-\ell_{k} j}=\sum_{k=1}^{N} \bar{C}_{k k} \bar{L}^{\ell_{\bar{k}} j} .
\end{aligned}
$$


Proof. The projection on $\mathfrak{g}_{+}$of $A=\sum_{k=1}^{N} C_{k k} L^{\ell_{k} j}, j>0$, is $\sum_{a \in \mathbb{S}_{+} \cup \mathbb{S}_{0}} B_{j \ell_{a}, a}$ while the projection on $\mathfrak{g}_{-}$of $A=\sum_{k=1}^{N} \bar{C}_{k k} \bar{L}^{-\ell_{\bar{k}} j}, j>0$, is $\sum_{a \in \overline{\mathfrak{S}}_{+}} B_{j \ell_{a}, a}$. The first formula is just $A=A_{+}+A_{-}$. The second formula follows in a similar way when $j<0$.

Now we proceed with

Proof of Proposition 5. Equations (43) and (44) follow from the previous lemma and Theorem 1 . To deduce (45) and (46) we argue as follows. If

$$
\sum_{a \in \mathcal{S}} \ell_{a}=0
$$

the periodicity follows from the factorization problem

$$
S \cdot W_{0} \cdot\left(\sum_{k=1}^{N} E_{k k} \Lambda^{\ell_{k}}\right) \cdot g=S \cdot W_{0} \cdot g \cdot\left(\sum_{k=1}^{N} E_{k k} \Lambda^{-\ell_{\bar{k}}}\right)=\bar{S} \cdot \bar{W}_{0} \cdot\left(\sum_{k=1}^{N} E_{k k} \Lambda^{-\ell_{\bar{k}}}\right)
$$

by observing that

$$
\begin{aligned}
& W_{0}\left(s_{1}+\ell_{1}, \ldots, s_{N}+\ell_{N}\right)=W_{0}\left(s_{1}, \ldots, s_{N}\right) \sum_{k=1}^{N} E_{k k} \Lambda^{\ell_{k}} \\
& \bar{W}_{0}\left(s_{\overline{1}}+\ell_{\overline{1}}, \ldots, s_{\bar{N}}+\ell_{\bar{N}}\right)=\bar{W}_{0}\left(s_{\overline{1}}, \ldots, s_{\bar{N}}\right) \sum_{k=1}^{N} E_{k k} \Lambda^{-\ell_{\bar{k}}}
\end{aligned}
$$

and recalling the uniqueness property of the factorization problem we deduce the periodicity condition for the solutions

$$
\begin{aligned}
& S\left(s_{1}+\ell_{1}, \ldots, s_{N}+\ell_{N}, s_{\overline{1}}+\ell_{\overline{1}}, \ldots, s_{\bar{N}}+\ell_{\bar{N}}\right)=S\left(s_{1}, \ldots, s_{N}, s_{\overline{1}}, \ldots, s_{\bar{N}}\right) \\
& \bar{S}\left(s_{1}+\ell_{1}, \ldots, s_{N}+\ell_{N}, s_{\overline{1}}+\ell_{\overline{1}}, \ldots, s_{\bar{N}}+\ell_{\bar{N}}\right)=\bar{S}\left(s_{1}, \ldots, s_{N}, s_{\overline{1}}, \ldots, s_{\bar{N}}\right)
\end{aligned}
$$

which imply (45) and (46).

Now we justify the name of this reduction. If we write $g=\sum_{j \in \mathbb{Z}} g_{j}(n) \Lambda^{j}$, and think of it as an element in $M_{N}\left(M_{\mathbb{Z}}(\mathbb{C})\right)$, i.e. $g=\sum_{k_{1}, k_{2}=1}^{N} g_{k_{1} k_{2}} E_{k_{1} k_{2}}$ and $g_{k_{1} k_{2}}=\sum_{j \in \mathbb{Z}} g_{j, k_{1} k_{2}}(n) \Lambda^{j}$ then (41) gives

$$
g_{j, k_{1} k_{2}}(n)=g_{j+\ell_{k_{1}}+\ell_{\bar{k}_{2}}, k_{1} k_{2}}\left(n-\ell_{k_{1}}\right) .
$$

If $\ell_{k_{1}}+\ell_{\bar{k}_{2}}=0$, then $g_{j, k_{1} k_{2}}$ is a $\left|\ell_{k_{1}}\right|$-periodic function in $n$. If this period is 1 , we get that $g_{k_{1} k_{2}}$ is a bi-infinite Toeplitz or Laurent matrix. We will see that in the general case we are dealing with block Toeplitz [5] and block Hankel [31] bi-infinite matrices.

Definition 4. Given a block matrix $\Omega=\left(\Omega_{i, j}\right)_{i, j \in \mathbb{Z}}$ made up with $p \times q$-blocks $\Omega_{i, j}$ we say that $\Omega$ is a block Toeplitz matrix if $\Omega_{i+1, j+1}=\Omega_{i, j}$ and a block Hankel matrix if $\Omega_{i+1, j-1}=\Omega_{i, j}$.

Proposition 6. The condition (48) implies for $g_{k_{1} k_{2}}$ that

- For $\ell_{k_{1}} \ell_{\bar{k}_{2}}>0$ is a $\left|\ell_{k_{1}}\right| \times\left|\ell_{\bar{k}_{2}}\right|$-block bi-infinite Hankel matrix.

- For $\ell_{k_{1}} \ell_{\bar{k}_{2}}<0$ is a $\left|\ell_{k_{1}}\right| \times\left|\ell_{\bar{k}_{2}}\right|$-block bi-infinite Toeplitz matrix.

- For $\ell_{k_{1}}=0$ with $\ell_{\bar{k}_{2}} \neq 0$ we have a diagonal band structure being $\left|\ell_{\bar{k}_{2}}\right|$ its width, and for $\ell_{\bar{k}_{2}}=0$ with $\ell_{k_{1}} \neq 0$ a $\left|\ell_{k_{1}}\right| \times\left|\ell_{k_{1}}\right|$ block bi-infinite matrix.

Proof. See Appendix B 
The Toeplitz/Hankel block structure appears not only in the structure of $g_{k_{1} k_{2}}$ but also in the structure of $g$ itself, thought as an element in $M_{\mathbb{Z}}\left(M_{N}(\mathbb{C})\right)$, for example if one takes $\ell_{k}=-\ell_{\bar{k}}=1, k=1, \ldots, N$ we get a $N \times N$-block bi-infinite Toeplitz matrix, while for $\ell_{k}=\ell_{\bar{k}}=1, k=1, \ldots, N$ we get a $N \times N$-block bi-infinite Hankel matrix.

Notice that for the particular case $\ell_{k}=\ell_{\bar{k}}, k=1, \ldots, N$, we have that $g$ is a block Hankel bi-infinite matrix and

$$
g \sum_{k=1}^{N} E_{k k} \Lambda^{-\ell_{k}}=\sum_{k=1}^{N} E_{k k} \Lambda^{\ell_{k}} g, \quad g \sum_{k=1}^{N} E_{k k} \Lambda^{\ell_{k}}=\sum_{k=1}^{N} E_{k k} \Lambda^{-\ell_{k}} g .
$$

From these two equivalent conditions on $g$ we conclude for $g^{2}$ the following constraint

$$
g^{2} \sum_{k=1}^{N} E_{k k} \Lambda^{\ell_{k}}=\sum_{k=1}^{N} E_{k k} \Lambda^{\ell_{k}} g^{2}
$$

i.e. $g^{2}$ is a block Toeplitz bi-infinite matrix and the corresponding solution to $g^{2}$ of periodic type with bared and non bared periods equal to each other for each component: $\ell_{\bar{k}}=-\ell_{k}, k=1, \ldots, N$.

In the one component case we get the condition

$$
L^{\ell_{1}}=\bar{L}^{-\ell_{\overline{1}}}
$$

If $\ell_{1}+\ell_{\overline{1}}=0$ we may choose $\ell_{1}=\ell \in \mathbb{N}$ and $\ell_{\overline{1}}=-\ell$ and the constraint for $g$ is $g \Lambda^{\ell}=\Lambda^{\ell} g$ which leads $L^{\ell}=\bar{L}^{\ell}$, i.e., the $\ell$-th periodic reduction of the one component $2 \mathrm{D}$ Toda hierarchy [30]. When $\ell_{1}, \ell_{\overline{1}}>0$ are two nonnegative integers this constraint (49) gives the the reduction of the one component 2D Toda hierarchy suitable to be extended with additional flows as described in [7], named there as bigraded. This is why we refer to this reduction when all $\ell_{a}$ are positive as multigraded reduction. Notice that this multigraded constraint over $g$ is never of periodic type and $\mathbb{S}=\mathbb{S}_{+}$and $\overline{\mathbb{S}}=\overline{\mathbb{S}}_{+}$.

$1 D$ reduction and generalizations Given a set of nonnegative integers $\left\{\ell_{a}\right\}_{a \in \mathcal{S}}$ we request $g$ in (9) the following constraint

$$
g \cdot\left(\sum_{k=1}^{N} E_{k k}\left(\Lambda^{\ell_{\bar{k}}}+\Lambda^{-\ell_{\bar{k}}}\right)\right)=\left(\sum_{k=1}^{N} E_{k k}\left(\Lambda^{\ell_{k}}+\Lambda^{-\ell_{k}}\right)\right) \cdot g
$$

Now, as $z^{j}+z^{-j}=\left(z+z^{-1}\right)^{j}+a_{j, j-2}\left(z+z^{-1}\right)^{j-2}+\cdots+a_{j, 0}$, for some $a_{j, i} \in \mathbb{Z}$ we have

$$
g \cdot\left(\sum_{k=1}^{N} E_{k k}\left(\Lambda^{j \ell_{\bar{k}}}+\Lambda^{-j \ell_{\bar{k}}}\right)\right)=\left(\sum_{k=1}^{N} E_{k k}\left(\Lambda^{j \ell_{k}}+\Lambda^{-j \ell_{k}}\right)\right) \cdot g
$$

and therefore

$$
\sum_{k=1}^{N} C_{k k}\left(L^{j \ell_{k}}+L^{-j \ell_{k}}\right)=\sum_{k=1}^{N} \bar{C}_{k k}\left(\bar{L}^{j \ell_{\bar{k}}}+\bar{L}^{-j \ell_{\bar{k}}}\right)
$$

is fulfilled for any $j \geqslant 0$.

From here we conclude that

$$
\sum_{k=1}^{N}\left(B_{j \ell_{k}, k}+B_{j \ell_{\bar{k}}, \bar{k}}\right)=\sum_{k=1}^{N} C_{k k}\left(L^{j \ell_{k}}+L^{-j \ell_{k}}\right)=\sum_{k=1}^{N} \bar{C}_{k k}\left(\bar{L}^{j \ell_{\bar{k}}}+\bar{L}^{-j \ell_{\bar{k}}}\right)
$$

and therefore we deduce the invariance

$$
\sum_{k=1}^{N}\left(\partial_{j \ell_{k}, k}+\partial_{j \ell_{\bar{k}}, \bar{k}}\right) L=\sum_{k=1}^{N}\left(\partial_{j \ell_{k}, k}+\partial_{j \ell_{\bar{k}}, \bar{k}}\right) \bar{L}=0 .
$$

In the one component case if we choose $\ell_{1}=\ell_{\overline{1}}=1$ we get the invariance under $\partial_{j 1}+\partial_{j \overline{1}}, j>0$. This is the 1 dimensional reduction as discussed for example in [30]. 
It must be stressed here that being the same invariance conditions (50) for this reduction and the previous multigraded reduction, the conditions are different for $g$ and therefore for the class of solutions considered in the 2D Toda hierarchy. In fact the Ueno-Takasaki 1D-reduction has soliton solutions, which appear as a particular class of the general soliton solutions of his 2D Toda hierarchy. However this Ueno-Takasaki's family of soliton solutions of 2D Toda does not admit the bigraded type condition. On the other hand, the condition $L=\bar{L}^{-1}$ appears as a string equation in 2D Toda leading to solutions of the 1-matrix models, see for example [25].

\section{Orlov-Schulman operators, undressing, and string equations}

\subsection{Introducing the Orlov-Schulman operator}

Given solutions $W, \bar{W}$ of the factorization problem (9) we introduce the Orlov-Schulman operators 23 for the multicomponent 2D Toda hierarchy

Definition 5. The Orlov-Schulman operators are defined as follows

$$
M:=W n W^{-1}, \quad \bar{M}:=\bar{W} n \bar{W}^{-1},
$$

Proposition 7. - The Orlov-Schulman operators satisfy the following commutation relations

$$
[L, M]=L, \quad\left[M, C_{k k}\right]=0, \quad[\bar{L}, \bar{M}]=\bar{L}, \quad\left[\bar{M}, \bar{C}_{k k}\right]=0,
$$

- The following relations hold

$$
\begin{array}{ll}
M=\mathcal{M}+\sum_{k=1}^{N} C_{k k}\left(s_{k}+\sum_{j=1}^{\infty} j t_{j k} L^{j}\right), \quad \mathcal{M}=n+\mathfrak{g}_{-} \\
\bar{M}=\overline{\mathcal{M}}-\sum_{k=1}^{N} \bar{C}_{k k}\left(s_{\bar{k}}+\sum_{j=1}^{\infty} j t_{j \bar{k}} \bar{L}^{-j}\right), & \overline{\mathcal{M}}=n+\mathfrak{g}_{+} \Lambda .
\end{array}
$$

Proof. See Appendix B.

Given the initial condition $g \in G$ in the factorization problem (9) we write

$$
g \Lambda E_{k k} g^{-1}=\sum_{l, l^{\prime}=1}^{N} p_{k, l l^{\prime}}(n, \Lambda) E_{l l^{\prime}}, \quad \quad g n E_{k k} g^{-1}=\sum_{l, l^{\prime}=1}^{N} q_{k, l l^{\prime}}(n, \Lambda) E_{l l^{\prime}}
$$

and define

$$
P_{k}:=\sum_{l, l^{\prime}=1}^{N} p_{k, l l^{\prime}}(M, L) C_{l l^{\prime}}, \quad Q_{k}=\sum_{l, l^{\prime}=1}^{N} q_{k, l l^{\prime}}(M, L) C_{l l^{\prime}}
$$

so that

$$
\left[P_{k}, Q_{k^{\prime}}\right]=\delta_{k k^{\prime}} P_{k}
$$

Then, as $W g=\bar{W}$, we get, in the language of [26], the string equations

$$
\sum_{l, l^{\prime}=1}^{N} p_{k, l l^{\prime}}(M, L) C_{l l^{\prime}}:=\bar{L} \bar{C}_{k k}, \quad \sum_{l, l^{\prime}=1}^{N} q_{k, l l^{\prime}}(M, L) C_{l l^{\prime}}:=\bar{M} \bar{C}_{k k} .
$$

\subsection{Undressing Lax equations for the Lax and Orlov-Schulman operators}

The Orlov-Schulman operators $M=W n W^{-1}, \bar{M}=\bar{W} n \bar{W}^{-1}$ satisfy

$$
\begin{aligned}
\partial_{j a} M & =\left[\partial_{j a} W \cdot W^{-1}, M\right], & & \partial_{j a} \bar{M}=\left[\partial_{j a} \bar{W} \cdot \bar{W}^{-1}, \bar{M}\right], \\
T_{K} M & =\left(T_{K} W \cdot W^{-1}\right) M\left(T_{K} W \cdot W^{-1}\right)^{-1}, & & T_{K} \bar{M}=\left(T_{K} \bar{W} \cdot \bar{W}^{-1}\right) \bar{M}\left(T_{K} \bar{W} \cdot \bar{W}^{-1}\right)^{-1}
\end{aligned}
$$


and the factorization problem (9) holds then the results of Theorem 11imply the following Lax equations for the Orlov-Schulman operators

$$
\begin{array}{ll}
\partial_{j a} M=\left[B_{j a}, M\right], & \partial_{j a} \bar{M}=\left[B_{j a}, \bar{M}\right], \\
T_{K} M=\omega_{K} M \omega_{K}^{-1}, & T_{K} \bar{M}=\omega_{K} \bar{M} \omega_{K}^{-1} .
\end{array}
$$

We now prove the local equivalence between the factorization problem and the Lax equations.

Theorem 2. Let us suppose that:

1. The operators $L, \bar{L}, C_{k k}, \bar{C}_{k k}, M$ and $\bar{M}$ satisfy the conditions

$$
\begin{aligned}
& L=\Lambda+u_{1}(n)+u_{2}(n) \Lambda^{-1}+\cdots, \quad \bar{L}^{-1}=\bar{u}_{0}(n) \Lambda^{-1}+\bar{u}_{1}(n)+\bar{u}_{2}(n) \Lambda+\cdots, \\
& C_{k k}=E_{k k}+C_{k k, 1}(n) \Lambda^{-1}+C_{k k, 2}(n) \Lambda^{-2}+\cdots, \quad \bar{C}_{k k}=\bar{C}_{k k, 0}(n)+\bar{C}_{k k, 1}(n) \Lambda+\bar{C}_{k k, 2}(n) \Lambda^{2}+\cdots, \\
& M=\cdots+M_{-1} \Lambda^{-1}+n \sum_{k=1}^{N} C_{k k}\left(s_{k}+\sum_{j=1}^{\infty} j t_{j k} L^{j}\right), \quad \bar{M}=\cdots+\bar{M}_{1} \Lambda+n+\sum_{k=1}^{N} \bar{C}_{k k}\left(s_{\bar{k}}+\sum_{j=1}^{\infty} j t_{j \bar{k}} \bar{L}^{-j}\right),
\end{aligned}
$$

with $k=1, \ldots, N, \bar{u}_{0}(n) \in \mathrm{GL}(N, \mathbb{C})$, and fulfill the equations

$$
\begin{aligned}
& \mathbb{\square}_{N}=\sum_{k=1}^{N} C_{k k}, \quad C_{k k} C_{l l}=\delta_{k l} C_{k k}, \quad C_{k k} L=L C_{k k}, \quad C_{k k} M=M C_{k k}, \quad L M=M L, \\
& \square_{N}=\sum_{k=1}^{N} \bar{C}_{k k}, \quad \bar{C}_{k k} \bar{C}_{l l}=\delta_{k l} \bar{C}_{k k}, \quad \bar{C}_{k k} \bar{L}=\bar{L} \bar{C}_{k k}, \quad \bar{C}_{k k} \bar{M}=\bar{M} \bar{C}_{k k}, \quad \bar{L} \bar{M}=\bar{M} \bar{L} .
\end{aligned}
$$

2. Given operators $B$ and $\omega$ as in (19), the Lax equations (29), (30) and (57) hold.

Then, there exists operators $S \in G_{-}$and $\bar{S} \in G_{+}$such that for $W=S W_{0}$ and $\bar{W}=\bar{S} \bar{W}_{0}$ we may write

$$
\begin{array}{lll}
L=W \Lambda W^{-1}, & M=W n W^{-1}, & C_{k k}=W E_{k k} W^{-1}, \\
\bar{L}=\bar{W} \Lambda \bar{W}^{-1}, & \bar{M}=\bar{W} n \bar{W}^{-1}, & \bar{C}_{k k}=\bar{W} E_{k k} \bar{W}^{-1},
\end{array}
$$

so that $W$ and $\bar{W}$ solve the factorization problem (9) for some constant operator $g$.

Notice that the set of constrains (58) and (59) are preserved by the Lax equations.

Proof. Observe thatwe need to find is the representation

$$
\begin{array}{lll}
L=S \Lambda S^{-1}, & M=S \mu S^{-1}, & C_{k k}=S E_{k k} S^{-1}, \\
\bar{L}=\bar{S} \Lambda \bar{S}^{-1}, & \bar{M}=\bar{S} \bar{\mu} \bar{S}^{-1}, & \bar{C}_{k k}=\bar{S} E_{k k} \bar{S}^{-1},
\end{array}
$$

with $\mu$ and $\bar{\mu}$ as in (89). We first undress the Lax operators $L, \bar{L}^{-1}$. We look for

$$
\begin{aligned}
& S^{\prime}=\mathbb{1}_{N}+\varphi_{1}^{\prime}(n) \Lambda^{-1}+\varphi_{2}^{\prime}(n) \Lambda^{-2}+\cdots, \quad \varphi_{j}^{\prime}: \mathbb{Z} \rightarrow M_{N}(\mathbb{C}), \\
& \bar{S}^{\prime}=\bar{\varphi}_{0}^{\prime}(n)\left(\mathbb{\square}_{N}+\bar{\varphi}_{1}^{\prime}(n) \Lambda+\bar{\varphi}_{2}^{\prime}(n) \Lambda^{2}+\cdots\right), \quad \bar{\varphi}_{0}^{\prime}: \mathbb{Z} \rightarrow \mathrm{GL}(N, \mathbb{C}), \quad \bar{\varphi}_{j}^{\prime}: \mathbb{Z} \rightarrow M_{N}(\mathbb{C}), j>0
\end{aligned}
$$

such that

$$
\begin{aligned}
& \left(\Lambda+u_{1}+u_{2} \Lambda^{-1}+\cdots\right)\left(\mathbb{\square}_{N}+\varphi_{1}^{\prime} \Lambda^{-1}+\varphi_{2}^{\prime} \Lambda^{-2}+\cdots\right)=\left(\mathbb{\square}_{N}+\varphi_{1}^{\prime} \Lambda^{-1}+\varphi_{2}^{\prime} \Lambda^{-2}+\cdots\right) \Lambda, \\
& \left(\bar{u}_{0} \Lambda^{-1}+\bar{u}_{1}+\bar{u}_{2} \Lambda^{2}+\cdots\right) \bar{\varphi}_{0}^{\prime}\left(\rrbracket_{N}+\bar{\varphi}_{1}^{\prime} \Lambda+\bar{\varphi}_{2}^{\prime} \Lambda^{2}+\cdots\right)=\bar{\varphi}_{0}^{\prime}\left(\rrbracket_{N}+\bar{\varphi}_{1}^{\prime} \Lambda+\bar{\varphi}_{2}^{\prime} \Lambda^{2}+\cdots\right) \Lambda^{-1} \text {. }
\end{aligned}
$$

Therefore, if we define $\mathbb{\Sigma}_{ \pm}[f]:=\sum_{j=0}^{\infty} f(n \pm j)$, we have

$$
\varphi_{1}^{\prime}=c_{1}+\mathbb{Z}_{+}\left[u_{1}\right]
$$

Once $\varphi_{1}^{\prime}$ is obtained we fix our attention in the second equation to get

$$
\varphi_{2}^{\prime}(n)=c_{2}+\mathbb{Z}_{+}\left[u_{2}(n)+u_{1} \varphi_{1}^{\prime}\right] .
$$


So forth and so on we get all the coefficients $\varphi_{j}^{\prime}$ up to integration constants $c_{j}$.

Now we analyze (61), which we write as follows

$$
\left(\bar{v}_{0} \Lambda^{-1}+\bar{v}_{1}+\bar{v}_{2} \Lambda^{2}+\cdots\right)\left(\rrbracket_{N}+\bar{\varphi}_{1}^{\prime} \Lambda+\bar{\varphi}_{2}^{\prime} \Lambda^{2}+\cdots\right)=\Lambda^{-1}+\bar{\varphi}_{1}^{\prime}+\bar{\varphi}_{2}^{\prime} \Lambda+\cdots
$$

with $\bar{v}_{j}(n):=\bar{\varphi}_{0}^{\prime}(n)^{-1} \bar{u}_{j}(n) \bar{\varphi}_{0}^{\prime}(n+j-1)$. From this we deduce that $\bar{v}_{0}=\mathbb{\square}_{N}$ or $\bar{u}_{0}(n)=\bar{\varphi}_{0}^{\prime}(n) \bar{\varphi}_{0}^{\prime}(n-1)^{-1}$. Denoting $\phi^{\prime}:=\log \bar{\varphi}_{0}^{\prime}$ we get $\log \bar{u}_{0}=\left(1-\Lambda^{-1}\right)\left(\phi^{\prime}\right)$ and therefore $\phi^{\prime}(n)=\exp \left(\bar{c}_{0}+\mathbb{\Sigma}_{-}\left[\log \bar{u}_{0}\right]\right)$ where $\bar{c}_{0}$ is a constant matrix. As $\bar{v}_{0}=\rrbracket_{N}$ we have

$$
\left(\Lambda^{-1}+\bar{v}_{1}+\bar{v}_{2} \Lambda^{2}+\cdots\right)\left(\rrbracket_{N}+\bar{\varphi}_{1}^{\prime} \Lambda+\bar{\varphi}_{2}^{\prime} \Lambda^{2}+\cdots\right)=\Lambda^{-1}+\bar{\varphi}_{1}^{\prime}+\bar{\varphi}_{2}^{\prime} \Lambda+\cdots
$$

which transmutes into (60) once we replace $u_{j}$ by $\bar{v}_{j}, \varphi_{j}^{\prime}$ by $\bar{\varphi}_{j}^{\prime}, j=1,2, \ldots$ and $\Lambda$ by $\Lambda^{-1}$. Thus, all the coefficients $\bar{\varphi}_{j}^{\prime}$ are expressed in terms of $\bar{v}_{j}$.

Now, we proceed to undress $C_{k k}$ and $\bar{C}_{k k}$

$$
C_{k k}^{\prime}:=S^{\prime-1} C_{k k} S^{\prime}, \quad \bar{C}_{k k}^{\prime}:=\bar{S}^{\prime-1} \bar{C}_{k k} \bar{S}^{\prime} .
$$

These operators commute with $\Lambda$, satisfy

$$
\begin{aligned}
& C_{k k}^{\prime}=E_{k k}+C_{k k, 1}^{\prime} \Lambda^{-1}+C_{k k, 2}^{\prime} \Lambda^{-2}+\cdots \\
& \bar{C}_{k k}^{\prime}=\bar{C}_{k k, 0}^{\prime}+\bar{C}_{k k, 1}^{\prime} \Lambda+\bar{C}_{k k, 2}^{\prime} \Lambda^{2}+\cdots
\end{aligned}
$$

and provide us with two different resolutions of the identity,

$$
\rrbracket_{N}=\sum_{k=1}^{N} C_{k k}^{\prime}, \quad C_{k k}^{\prime} C_{l l}^{\prime}=\delta_{k l} C_{k k}^{\prime}, \quad \square_{N}=\sum_{k=1}^{N} \bar{C}_{k k}^{\prime}, \quad \bar{C}_{k k}^{\prime} \bar{C}_{l l}^{\prime}=\delta_{k l} \bar{C}_{k}^{\prime} .
$$

In fact, it is easy to show that there exists operators $Q=\rrbracket_{N}+Q_{1} \Lambda^{-1}+\cdots \in G_{-} \cap \bigcap_{\Lambda}$ and $\bar{Q}=\bar{Q}_{0}+\bar{Q}_{1} \Lambda+\cdots \in$ $G_{+} \cap \mathfrak{z}_{\Lambda}$ such that $C_{k k}^{\prime}=Q E_{k k} Q^{-1}$ and $\bar{C}_{k k}^{\prime}=\bar{Q} E_{k k} \bar{Q}^{-1}$. Thus, to undress $L, \bar{L}, C_{k k}, \bar{C}_{k k}, k=1, \ldots, N$, we just take $S=S^{\prime} \cdot Q, \bar{S}=\bar{S}^{\prime} \cdot \bar{Q}$.

With these operators at hand we proceed to undress $M$ and $\bar{M}$

$$
S^{-1} M S=\alpha+\mu, \quad \alpha:=S^{-1} \mathcal{M} S-n, \quad \bar{S}^{-1} \bar{M} \bar{S}=\bar{\alpha}+\bar{\mu}, \quad \bar{\alpha}:=\bar{S}^{-1} \overline{\mathcal{M}} \bar{S}-n,
$$

but

$$
\left[\Lambda, S^{-1} M S\right]=\Lambda, \quad\left[E_{k k}, S^{-1} M S\right]=0, \quad\left[\Lambda, \bar{S}^{-1} \bar{M} \bar{S}\right]=\Lambda, \quad\left[E_{k k}, \bar{S}^{-1} \bar{M} \bar{S}\right]=0
$$

and $[\Lambda, \mu]=\Lambda$ and $[\Lambda, \bar{\mu}]=\Lambda$, so that

$$
[\Lambda, \alpha]=0, \quad\left[E_{k k}, \alpha\right]=0, \quad[\Lambda, \bar{\alpha}]=0, \quad\left[E_{k k}, \bar{\alpha}\right]=0 \quad \Rightarrow \quad \alpha, \bar{\alpha} \in \mathfrak{h} .
$$

Now, recalling that $\mathcal{M}=n+\mathfrak{g}_{-}$and $\overline{\mathcal{M}}=n+\Lambda \mathfrak{g}_{+}$we write $\alpha=S^{-1} n S-n+\mathfrak{g}_{-}$and $\bar{\alpha}=\bar{S}^{-1} n \bar{S}-n+\mathfrak{g}_{+} \Lambda$ so that $\alpha=\alpha_{1} \Lambda^{-1}+\alpha_{2} \Lambda^{-2}+\cdots$ and $\bar{\alpha}=\bar{\alpha}_{1} \Lambda+\bar{\alpha}_{2} \Lambda^{2}+\cdots$ with $\alpha_{i}, \bar{\alpha}_{i} \in \operatorname{diag}(N, \mathbb{C})$ for all $i \in \mathbb{N}$. We define $\gamma:=-\sum_{j \geqslant 1} \frac{\alpha_{j}}{j} \Lambda^{-j}, \bar{\gamma}:=\phi_{0}+\sum_{j \geqslant 1} \frac{\bar{\alpha}_{j}}{j} \Lambda^{j}$, where $\phi_{0} \in \operatorname{diag}(N, \mathbb{C})$, and find that

$$
\mathrm{e}^{\gamma} n \mathrm{e}^{-\gamma}=n+[\gamma, n]=n+\alpha=S^{-1} \mathcal{M} S, \quad \mathrm{e}^{\bar{\gamma}} n \mathrm{e}^{-\bar{\gamma}}=n+[\bar{\gamma}, n]=n+\bar{\alpha}=\bar{S}^{-1} \overline{\mathcal{M}} \bar{S}
$$

which allows us to write

$$
\mathrm{e}^{\gamma} W_{0} n W_{0}^{-1} \mathrm{e}^{-\gamma}=\mathrm{e}^{\gamma} n \mathrm{e}^{-\gamma}+\nu=S^{-1} \mathcal{M} S+\nu, \quad \mathrm{e}^{\bar{\gamma}} \bar{W}_{0} n \bar{W}_{0}^{-1} \mathrm{e}^{-\bar{\gamma}}=\mathrm{e}^{\bar{\gamma}} n \mathrm{e}^{-\bar{\gamma}}+\bar{\nu}=\bar{S}^{-1} \overline{\mathcal{M}} \bar{S}+\bar{\nu} .
$$

Therefore, if we replace $S \rightarrow S \mathrm{e}^{\gamma}$ and $\bar{S} \rightarrow \bar{S} \mathrm{e}^{\bar{\gamma}}$, we get the desired result.

From the evolution equation we get that

$$
\begin{array}{rlrl}
A_{j a} & :=(S)^{-1} \cdot\left(B_{j a}-\partial_{j a} S \cdot(S)^{-1}\right) S, & \bar{A}_{j a} & :=(\bar{S})^{-1} \cdot\left(B_{j a}-\partial_{j a} \bar{S} \cdot(\bar{S})^{-1}\right) \bar{S}, \\
\rho_{K}:=T_{K}(S)^{-1} \omega_{K} S, & \bar{\rho}_{K}:=T_{K}(\bar{S})^{-1} \omega_{K} \bar{S},
\end{array}
$$


commute with $\Lambda$ and all the $E_{k k}, k=1, \ldots, N$; i.e., they are $n$-independent and diagonal.We may deduce that

$$
\begin{aligned}
& {\left[A_{j n}, \mu\right]=j \theta_{j a} \Rightarrow\left[A_{j a}, n\right]=j \theta_{j a} \Rightarrow\left[A_{j a}-\theta_{j a}, n\right]=0,} \\
& {\left[\bar{A}_{j n}, \bar{\mu}\right]=-j \bar{\theta}_{j a} \Rightarrow\left[\bar{A}_{j a}, n\right]=-j \bar{\theta}_{j a} \Rightarrow\left[\bar{A}_{j a}-\bar{\theta}_{j a}, n\right]=0,}
\end{aligned}
$$

and

$$
\begin{aligned}
& \omega_{K} M \omega_{K}^{-1}=\left(T_{K} S \cdot S^{-1}\right) M\left(T_{K} S \cdot S^{-1}\right)^{-1}+\left(T_{K} S\right)\left(\pi_{a}-\pi_{b}\right)\left(T_{K} S\right)^{-1}, \\
& \bar{\omega}_{K} \bar{M} \bar{\omega}_{K}^{-1}=\left(T_{K} \bar{S} \cdot \bar{S}^{-1}\right) \bar{M}\left(T_{K} \bar{S} \cdot S^{-1}\right)^{-1}-\left(T_{K} \bar{S}\right)\left(\bar{\pi}_{a}-\bar{\pi}_{b}\right)\left(T_{K} \bar{S}\right)^{-1},
\end{aligned}
$$

which imply

$$
\begin{aligned}
& {\left[\rho_{k}, \mu\right]=\left(\pi_{a}-\pi_{b}\right) \rho_{k} \Rightarrow\left[\rho_{k}, n\right]=\left(\pi_{a}-\pi_{b}\right) \rho_{k} \Rightarrow\left[\rho_{k} q_{K}^{-1}, n\right]=0,} \\
& {\left[\bar{\rho}_{k}, \bar{\mu}\right]=-\left(\bar{\pi}_{a}-\bar{\pi}_{b}\right) \bar{\rho}_{k} \Rightarrow\left[\bar{\rho}_{k}, n\right]=-\left(\bar{\pi}_{a}-\bar{\pi}_{b}\right) \bar{\rho}_{k} \Rightarrow\left[\bar{\rho}_{k} \bar{q}_{K}^{-1}, n\right]=0 .}
\end{aligned}
$$

Thus,

$$
A_{j a}-\theta_{j a}, \bar{A}_{j a}-\bar{\theta}_{j a}, \rho_{k} q_{K}^{-1}, \bar{\rho}_{k} \bar{q}_{K}^{-1} \in \operatorname{diag}(N, \mathbb{C}) .
$$

As the Lax equations (29) and (30) are satisfied by Proposition 4 we know that $B_{j a}$ and $\omega_{K}$ satisfy the compatibility conditions (31)-(37). However, we see from (62) that $\left\{A_{j a}, \rho_{K}\right\}$ and $\left\{\bar{A}_{j a}, \bar{\rho}_{K}\right\}$ are gauge transforms of $B_{j a}, \omega_{K}$ and thereby do have zero curvature. Therefore, we conclude the local existence of potentials $\xi$ and $\bar{\xi}$ such that

$$
A_{j a}=\partial_{j a} \xi \cdot \xi^{-1}, \quad \bar{A}_{j a}=\partial_{j a} \bar{\xi} \cdot \bar{\xi}^{-1}, \quad \rho_{K}=T_{K} \xi \cdot \xi^{-1}, \quad \bar{\rho}_{K}=T_{K} \bar{\xi} \cdot \bar{\xi}^{-1} .
$$

These potentials are determined up to right multiplication $\xi \rightarrow \xi \cdot h, \quad \bar{\xi} \rightarrow \bar{\xi} \cdot \bar{h}$, where $h, \bar{h} \in G$ are constant operators independent of $t_{j a}, s_{a}$. Up to this freedom we may take the potentials $\xi, \bar{\xi} \in H$.Now, recalling (19) we get

$$
B_{j a}=\mathcal{R}_{j a}+\mathfrak{g}_{-}=\overline{\mathcal{R}}_{j a}+\mathfrak{g}_{+} \quad \omega_{K}=G_{-} \cdot \mathcal{U}_{K}=G_{+} \cdot \overline{\mathcal{U}}_{K},
$$

which together with (62) imply $A_{j a}-\theta_{j a} \in \mathfrak{g}_{-}, \bar{A}_{j a}-\bar{\theta}_{j a} \in \mathfrak{g}_{+}, \rho_{K} q_{K}^{-1} \in G_{-}, \bar{\rho}_{K} \bar{q}_{K}^{-1} \in G_{+}$; but these operators belong to $\operatorname{diag}(N, \mathbb{C})$. Thus, from the two first relations we conclude $A_{j a}=\theta_{j a}, \rho_{K}=q_{K}$ and $\xi=W_{0}$ while the two second imply that if $\bar{\xi}=\mathrm{e}^{\phi_{0}} \cdot \bar{W}_{0}$ then $\phi_{0} \in \operatorname{diag}(N, \mathbb{C})$.

Therefore, we may write

$$
A_{j a}=\partial_{j a} W_{0} \cdot W_{0}^{-1}, \quad \bar{A}_{j a}=\partial_{j a}\left(\bar{\xi} \bar{W}_{0}\right) \cdot\left(\mathrm{e}^{\phi_{0}} \bar{W}_{0}\right)^{-1}, \quad \rho_{K}=T_{K} W_{0} \cdot W_{0}^{-1}, \quad \bar{\rho}_{K}=T_{K}\left(\mathrm{e}^{\phi_{0}} \bar{W}_{0}\right) \cdot\left(\mathrm{e}^{\phi_{0}} \bar{W}_{0}\right)^{-1} .
$$

We make the replacement $\bar{S} \rightarrow \bar{S} \mathrm{e}^{\phi_{0}}$ to get

$$
\begin{gathered}
B_{j a}=\partial_{j a} W \cdot W^{-1}=\partial_{j a} S \cdot S^{-1}+S \theta_{j a} S^{-1}=\partial_{j a} \bar{S} \cdot \bar{S}^{-1}+\bar{S} \bar{\theta}_{j a} \bar{S}^{-1}=\partial_{j a} \bar{W} \cdot \bar{W}^{-1}, \\
\omega_{K}=\left(T_{K} W\right) \cdot W^{-1}=\left(T_{K} S\right) q_{k} S^{-1}=\left(T_{K} \bar{S}\right) \bar{q}_{K} \bar{S}^{-1}=\left(T_{K} \bar{W}\right) \cdot \bar{W}^{-1}
\end{gathered}
$$

In terms of $g=W^{-1} \cdot \bar{W}$ the previous equations can be written as $\partial_{j a} g=0$ and $T_{K} g=g$. Thus, we finally find $W g=\bar{W}$ where $g$ is a constant operator in $G$.

A further result regarding the operators $C_{k l}$ introduced in Definition 2 and characterized in Proposition 1 that will be needed later is given now.

Proposition 8. Given operators $L, \bar{L}, M, \bar{M}, C_{k k}$ and $\bar{C}_{k k}$ as in Theorem Q then: if we find operators $C_{k l}$ of the form

$$
C_{k l}=L^{s_{k}-s_{l}} \mathrm{e}^{\left(t_{j k}-t_{j l}\right) L^{j}}\left(E_{k l}+\mathfrak{g}_{-}\right)
$$

such that

$$
\left[C_{k l}, L\right]=\left[C_{k l}, M\right]=0, \quad \quad C_{k^{\prime} k^{\prime}} C_{k l}=\delta_{k k^{\prime}} C_{k^{\prime} l}, \quad C_{k l} C_{k^{\prime} k^{\prime}}=\delta_{k l^{\prime}} C_{k k^{\prime}},
$$

then the undressing operator $W$ of Theorem 0 satisfies $C_{k l}=W E_{k l} W^{-1}$.

Proof. See Appendix B. 


\subsection{String equations, factorization problem and Lax equations}

We will show here that the string equations (56) for the Lax and Orlov-Schulman operators do indeed imply the factorization (9) and also the Lax equations (29)-(30). In fact, only one of these implications is needed as the other one will follow from the results described previously. However, we show that these two facts can be derived directly from the string equations, showing the importance of this formulation of integrable systems.

Theorem 3. Let $L, M, C_{k k}, \bar{L}, \bar{M}, \bar{C}_{k k}, k=1, \ldots, N$, be operators as in Theorem 0 and operators $C_{k l}, k, l=$ $1, \ldots, N$, be as in Proposition 8. Let us suppose that we have operators $P_{k}, Q_{k}$ as in (54) and that the string equations (56) hold. Then,

1. We can choose the operators $W$ and $\bar{W}$ of Theorem Q such that the factorization (9) holds for some constant operator $g \in G$.

2. The Lax equations (29)-(30) are fulfilled.

Proof. From the first part of the proof of Theorem 2 (not considering the Lax equations) and Proposition 8 we know that there are undressing operators $W=S W_{0}$ and $\bar{W}=\bar{S} \bar{W}_{0}, S \in G_{-}$and $\bar{S} \in G_{+}$. Let us introduce some convenient notation

$$
\begin{aligned}
D_{j a} & :=\partial_{j a} W \cdot W^{-1}-\partial_{j a} \bar{W} \cdot \bar{W}^{-1} & D_{j a}^{0}: & :=\bar{W}^{-1} D_{j a} \bar{W}, \\
\sigma_{K} & :=\left(T_{K} \bar{W} \cdot \bar{W}^{-1}\right)^{-1} T_{K} W \cdot W^{-1} & \sigma_{K}^{0} & :=\bar{W}^{-1} \sigma_{K} \bar{W},
\end{aligned}
$$

and observe that if we define

$$
\zeta:=\bar{W}^{-1} \cdot W
$$

we have

$$
D_{j a}^{0}=\partial_{j a} \zeta \cdot \zeta^{-1}, \quad \sigma_{K}^{0}=T_{K} \zeta \cdot \zeta^{-1} .
$$

The string equations (56) read

$$
\begin{aligned}
& P_{k}=W P_{k}^{0} W^{-1}=\bar{W} E_{k k} \Lambda \bar{W}^{-1}, \quad P_{k}^{0}=\sum_{l, l^{\prime}=1}^{N} p_{k, l l^{\prime}}(n, \Lambda) E_{l l^{\prime}}, \\
& Q_{k}=W Q_{k}^{0} W^{-1}=\bar{W} E_{k k} n \bar{W}^{-1}, \quad Q_{k}^{0}=\sum_{l, l^{\prime}=1}^{N} q_{k, l l^{\prime}}(n, \Lambda) E_{l l^{\prime}},
\end{aligned}
$$

which in turn imply

$$
\begin{aligned}
\partial_{j a} P_{k} & =\left[\partial_{j a} W \cdot W^{-1}, P_{k}\right]=\left[\partial_{j a} \bar{W} \cdot \bar{W}^{-1}, P_{k}\right], \\
\partial_{j a} Q_{k} & =\left[\partial_{j a} W \cdot W^{-1}, Q_{k}\right]=\left[\partial_{j a} \bar{W} \cdot \bar{W}^{-1}, Q_{k}\right] \\
T_{K} P_{k} & =\left(T_{K} W \cdot W^{-1}\right) P_{k}\left(T_{K} W \cdot W^{-1}\right)^{-1}=\left(T_{K} \bar{W} \cdot \bar{W}^{-1}\right) P_{k}\left(T_{K} \bar{W} \cdot \bar{W}^{-1}\right)^{-1} \\
T_{K} Q_{k} & =\left(T_{K} W \cdot W^{-1}\right) Q_{k}\left(T_{K} W \cdot W^{-1}\right)^{-1}=\left(T_{K} \bar{W} \cdot \bar{W}^{-1}\right) Q_{k}\left(T_{K} \bar{W} \cdot \bar{W}^{-1}\right)^{-1} .
\end{aligned}
$$

Hence, recalling $P_{k}=\bar{L} \bar{C}_{k k}$ and $Q_{k}=\bar{M} \bar{C}_{k k}$ we conclude that

$$
\left[D_{j a}, \bar{L}\right]=\left[D_{j a}, \bar{M}\right]=\left[D_{j a}, \bar{C}_{k k}\right]=0, \quad\left[\sigma_{K}, \bar{L}\right]=\left[\sigma_{K}, \bar{M}\right]=\left[\sigma_{K}, \bar{C}_{k k}\right]=0
$$

Thus, we deduce that $D_{j a}^{0}, \sigma_{K}^{0} \in \operatorname{diag}(N, \mathbb{C})$, and therefore $D_{j a} \in \mathfrak{g}_{+}$and $\sigma_{K} \in G_{+}$. With these preliminaries let us start proving the two statements in the theorem

1. Given the representation (69) in terms of $\zeta$ as in (68) we deduce that we can write $\zeta=\bar{\xi} \cdot g^{-1}$ for some $\bar{\xi} \in \operatorname{diag}(N, \mathbb{C})$ and some $(\boldsymbol{s}, \boldsymbol{t})$ - independent operator $g \in G$. Thus, $D_{j a}^{0}=\partial_{j a} \bar{\xi} \cdot \bar{\xi}^{-1}$ and $\sigma_{K}^{0}=T_{K} \bar{\xi} \cdot \bar{\xi}^{-1}$. But, recalling the definition (68) we get $\bar{W} \zeta=W \Rightarrow \bar{S} \bar{W}_{0} \bar{\xi}=W g$. Observe that $\left[\bar{W}_{0}, \bar{\xi}\right]=0$ and replace $\bar{S} \rightarrow \bar{S} \cdot \bar{\xi}$ to get the factorization problem. 
2. From the definition (67) we get

$$
\begin{aligned}
& \partial_{j a} W \cdot W^{-1}=\partial_{j a} S \cdot S^{-1}+\mathcal{R}_{j a}=\partial_{j a} \bar{S} \cdot \bar{S}^{-1}+\overline{\mathcal{R}}_{j a}+D_{j a}=\partial_{j a} \bar{W} \cdot \bar{W}^{-1}+D_{j a} \\
& T_{K} W \cdot W^{-1}=T_{K} S \cdot S^{-1} \cdot \mathcal{U}_{K}=T_{K} \bar{S} \cdot \bar{S}^{-1} \cdot \overline{\mathcal{U}}_{K} \cdot \sigma_{K}=T_{K} \bar{W} \cdot \bar{W}^{-1} \cdot \sigma_{K}
\end{aligned}
$$

Reasoning as in the proof of Theorem 2 and recalling that $D_{j a} \in \mathfrak{g}_{+}, \sigma_{K} \in G_{+}$and $\left[\overline{\mathcal{U}}_{K}, \sigma_{K}\right]=0$ we have

$$
\begin{aligned}
& \partial_{j a} S \cdot S^{-1}=-\left(\mathcal{R}_{j a}-\overline{\mathcal{R}}_{j a}\right)_{-}, \\
& \partial_{j a} \bar{S} \cdot \bar{S}^{-1}+D_{j a}=\left(\mathcal{R}_{j a}-\overline{\mathcal{R}}_{j a}\right)_{+}, \\
& T_{K} S \cdot S^{-1}=\left(\mathcal{U}_{K} \cdot \overline{\mathcal{U}}_{K}^{-1}\right)_{-}, \\
& T_{K} \bar{S} \cdot \bar{S}^{-1} \cdot \sigma_{K}=\left(\mathcal{U}_{K} \cdot \overline{\mathcal{U}}_{K}^{-1}\right)_{+}
\end{aligned}
$$

so that, according to (19),

$$
\partial_{j a} W \cdot W^{-1}=B_{j a}=\partial_{j a} \bar{W} \cdot \bar{W}^{-1}+D_{j a}, \quad T_{K} W \cdot W^{-1}=\omega_{K}=T_{K} \bar{W} \cdot \bar{W}^{-1} \cdot \sigma_{K} \cdot
$$

Therefore, we immediately get the following Lax equations

$$
\begin{array}{rlrl}
\partial_{j a} L & =\left[\partial_{j a} W \cdot W^{-1}, L\right]=\left[B_{j a}, L\right], & T_{K} L & =\left(T_{K} W \cdot W^{-1}\right) L\left(T_{K} W \cdot W^{-1}\right)^{-1}=\omega_{K} L \omega_{K}^{-1} \\
\partial_{j a} M & =\left[\partial_{j a} W \cdot W^{-1}, M\right]=\left[B_{j a}, M\right], & T_{K} M=\left(T_{K} W \cdot W^{-1}\right) M\left(T_{K} W \cdot W^{-1}\right)^{-1}=\omega_{K} M \omega_{K}^{-1} \\
\partial_{j a} C_{k k} & =\left[\partial_{j a} W \cdot W^{-1}, C_{k k}\right]=\left[B_{j a}, C_{k k}\right], & T_{K} C_{k k} & =\left(T_{K} W \cdot W^{-1}\right) C_{k k}\left(T_{K} W \cdot W^{-1}\right)^{-1}=\omega_{K} C_{k k} \omega_{K}^{-1} .
\end{array}
$$

Now, as $\partial_{j a} \bar{W} \cdot \bar{W}^{-1}=B_{j a}-D_{j a}$ and $T_{K} \bar{W} \cdot \bar{W}^{-1}=\omega_{K} \cdot \sigma_{K}$ with $D_{j a}$ and $\sigma_{K}$ commuting with any function of $\bar{L}, \bar{M}$ and $\bar{C}_{k k}$ we get the remaining Lax equations

$$
\begin{array}{rlrl}
\partial_{j a} \bar{L} & =\left[\partial_{j a} \bar{W} \cdot \bar{W}^{-1}, \bar{L}\right]=\left[B_{j a}, \bar{L}\right], & T_{K} \bar{L} & =\left(T_{K} \bar{W} \cdot \bar{W}^{-1}\right) \bar{L}\left(T_{K} \bar{W} \cdot \bar{W}^{-1}\right)^{-1}=\omega_{K} \bar{L} \omega_{K}^{-1} \\
\partial_{j a} \bar{M} & =\left[\partial_{j a} \bar{W} \cdot \bar{W}^{-1}, \bar{M}\right]=\left[B_{j a}, M\right], & T_{K} \bar{M}=\left(T_{K} \bar{W} \cdot \bar{W}^{-1}\right) \bar{M}\left(T_{K} \bar{W} \cdot \bar{W}^{-1}\right)^{-1}=\omega_{K} \bar{M} \omega_{K}^{-1} \\
\partial_{j a} \bar{C}_{k k} & =\left[\partial_{j a} \bar{W} \cdot \bar{W}^{-1}, \bar{C}_{k k}\right]=\left[B_{j a}, C_{k k}\right], & T_{K} \bar{C}_{k k}=\left(T_{K} \bar{W} \cdot W^{-1}\right) \bar{C}_{k k}\left(T_{K} \bar{W} \cdot \bar{W}^{-1}\right)^{-1}=\omega_{K} \bar{C}_{k k} \omega_{K}^{-1} .
\end{array}
$$

The above result might be slightly generalized by considering string equations of the form

$$
\sum_{l, l^{\prime}=1}^{N} p_{k, l l^{\prime}}(M, L) C_{l l^{\prime}}=\sum_{l, l^{\prime}=1}^{N} \bar{p}_{k, l l^{\prime}}(\bar{M}, \bar{L}) \bar{C}_{l l^{\prime}}, \quad \sum_{l, l^{\prime}=1}^{N} q_{k, l l^{\prime}}(M, L) C_{l l^{\prime}}=\sum_{l, l^{\prime}=1}^{N} \bar{q}_{k, l l^{\prime}}(\bar{M}, \bar{L}) \bar{C}_{l l^{\prime}}
$$

where we assume that

$$
\bar{P}_{k}^{0}:=\sum_{l, l^{\prime}=1}^{N} \bar{p}_{k, l l^{\prime}}(n, \Lambda) \bar{E}_{l l^{\prime}}, \quad \quad \bar{Q}_{k}^{0}:=\sum_{l, l^{\prime}=1}^{N} \bar{q}_{k, l l^{\prime}}(n, \Lambda) \bar{E}_{l l^{\prime}}, \quad k=1, \ldots, N
$$

belong to the adjoint orbit $\mathscr{O}$ of $E_{k k} \Lambda, E_{k k} n, k=1, \ldots, N$; i.e., there exists $\bar{g} \in G$ such that

$$
P_{k}^{0}=\bar{g} \cdot E_{k k} \Lambda \cdot \bar{g}^{-1}, \quad Q_{k}^{0}=\bar{g} \cdot E_{k k} n \cdot \bar{g}^{-1} .
$$

For that aim the proof needs to be modified only in the definition of $D_{j a}^{0} \rightarrow \bar{g}^{-1} D_{j a}^{0} \bar{g}^{-1}$ and $\sigma_{K}^{0} \rightarrow \bar{g}^{-1} \sigma_{K}^{0} \bar{g}$ and $g \rightarrow g \cdot \bar{g}$. Observe that elements in $\mathscr{O}$ can be constructed in terms of operators $\mathcal{C}_{k}, \mathcal{P}$ and $\mathcal{Q}$, such that:

$$
\sum_{k=1}^{N} \mathcal{C}_{k}=\rrbracket_{N}, \quad \mathcal{C}_{k} \mathcal{C}_{k^{\prime}}=\delta_{k k^{\prime}} \mathcal{C}_{k}, \quad\left[\mathcal{C}_{k}, \mathcal{P}\right]=\left[\mathcal{C}_{k}, \mathcal{Q}\right]=0, \quad[\mathcal{P}, \mathcal{Q}]=\mathcal{P}
$$

What we do not know yet is if the orbit $\mathscr{O}$ is characterized precisely by this properties. However, if we request the following properties: $\mathcal{C}_{k}-E_{k k} \in \mathfrak{g}_{-}, \mathcal{P}-\Lambda \in \mathfrak{g}_{-} \Lambda, \mathcal{Q}-n \in \mathfrak{g}_{-}$, one could prove, following similar arguments as in the proof of Theorem 2, that these elements lay in $\mathscr{O}$. This implies an alternative formulation of string equations (56)

$$
\sum_{l, l^{\prime}} \mathcal{C}_{k, l l^{\prime}}(L, M) C_{l l^{\prime}}=\bar{C}_{k k}, \quad \sum_{l, l^{\prime}} \mathcal{P}_{l l^{\prime}}(L, M) C_{l l^{\prime}}=\bar{L}, \quad \sum_{l, l^{\prime}} \mathcal{Q}_{l l^{\prime}}(L, M) C_{l l^{\prime}}=\bar{M} .
$$




\subsection{Additional symmetries and string equations}

\subsubsection{Additional symmetries}

Suppose that the operator $g$ in (9) depends on an additional, or external, parameter $b$, which might belong to $\mathbb{C}$ or to $\mathbb{Z}$. Now, we will describe the induced dependence on the elements defining the multicomponent Toda hierarchy. We shall denote by $\partial_{6}=\partial / \partial 6$ when $\sigma \in \mathbb{C}$ is a continuous an by $T_{6}$ the corresponding shift $6 \rightarrow 6+1$, when $6 \in \mathbb{Z}$ is an integer. In this case, we shall replace (9) by the equivalent factorization problem $W \cdot h=\bar{W} \cdot \bar{h}$, with

$$
g=h \cdot \bar{h}^{-1} .
$$

Observe that for $6 \in \mathbb{C}$ we may write,

$$
\begin{aligned}
\partial_{6} W \cdot W^{-1}+W\left(\partial_{6} h \cdot h^{-1}\right) W^{-1} & =\partial_{6} S \cdot S^{-1}+W\left(\partial_{6} h \cdot h^{-1}\right) W^{-1} \\
& =\partial_{6} \bar{S} \cdot \bar{S}^{-1}+\bar{W}\left(\partial_{6} \bar{h} \cdot \bar{h}^{-1}\right) \bar{W}^{-1}=\partial_{6} \bar{W} \cdot \bar{W}^{-1}+\bar{W}\left(\partial_{6} \bar{h} \cdot \bar{h}^{-1}\right) \bar{W}^{-1},
\end{aligned}
$$

while for $6 \in \mathbb{Z}$ we have

$$
\begin{aligned}
T_{6} W \cdot W^{-1} \cdot W \cdot\left(T_{6} h \cdot h^{-1}\right) \cdot W^{-1} & =T_{6} S \cdot S^{-1} \cdot W \cdot\left(T_{6} h \cdot h^{-1}\right) \cdot W^{-1} \\
& =T_{6} \bar{S} \cdot \bar{S}^{-1} \cdot \bar{W} \cdot\left(T_{6} \bar{h} \cdot \bar{h}^{-1}\right) \cdot \bar{W}^{-1}=T_{6} \bar{W} \cdot \bar{W}^{-1} \cdot \bar{W} \cdot\left(T_{6} \bar{h} \cdot \bar{h}^{-1}\right) \cdot \bar{W}^{-1}
\end{aligned}
$$

Now we suppose that the dependence on 6 is given by the following equations

$$
\begin{array}{ll}
\partial_{6} h \cdot h^{-1}=F_{0}=\sum_{l, l^{\prime}=1}^{N} F_{l l^{\prime}}(n, \Lambda) E_{l l^{\prime}}, \quad \partial_{b} \bar{h} \cdot \bar{h}^{-1}=\bar{F}_{0}=\sum_{l, l^{\prime}=1}^{N} \bar{F}_{l l^{\prime}}(n, \Lambda) E_{l l^{\prime}} \quad \text { when } 6 \in \mathbb{C}, \\
T_{6} h \cdot h^{-1}=\mathcal{F}_{0}=\sum_{l, l^{\prime}=1}^{N} \mathcal{F}_{l l^{\prime}}(n, \Lambda) E_{l l^{\prime}}, \quad T_{6} \bar{h} \cdot \bar{h}^{-1}=\overline{\mathcal{F}}_{0}=\sum_{l, l^{\prime}=1}^{N} \overline{\mathcal{F}}_{l l^{\prime}}(n, \Lambda) E_{l l^{\prime}}, \quad & \text { when } 6 \in \mathbb{Z},
\end{array}
$$

and define

$$
\begin{aligned}
& F:=\sum_{l, l^{\prime}=1}^{N} F_{l l^{\prime}}(M, L) C_{l l^{\prime}}, \quad \bar{F}:=\sum_{l, l^{\prime}=1}^{N} \bar{F}_{l l^{\prime}}(\bar{M}, \bar{L}) \bar{C}_{l l^{\prime}} \quad \text { when } 6 \in \mathbb{C}, \\
& \mathcal{F}:=\sum_{l, l^{\prime}=1}^{N} \mathcal{F}_{l l^{\prime}}(M, L) C_{l l^{\prime}}, \quad \overline{\mathcal{F}}:=\sum_{l, l^{\prime}=1}^{N} \overline{\mathcal{F}}_{l l^{\prime}}(\bar{M}, \bar{L}) \bar{C}_{l l^{\prime}}, \quad \text { when } 6 \in \mathbb{C} .
\end{aligned}
$$

From (73)-(174) we get

$$
\begin{array}{lll}
\partial_{6} W \cdot W^{-1}=\partial_{6} S \cdot S^{-1}=-(F-\bar{F})_{-}, & \partial_{6} \bar{W} \cdot \bar{W}^{-1}=\partial_{6} \bar{S} \cdot \bar{S}^{-1}=(F-\bar{F})_{+}, & (F-\bar{F})_{ \pm} \in \mathfrak{g}_{ \pm}, \\
T_{6} W \cdot W^{-1}=T_{6} S \cdot S^{-1}=\left(\mathcal{F} \cdot \overline{\mathcal{F}}^{-1}\right)_{-}, & T_{6} \bar{W} \cdot \bar{W}^{-1}=T_{6} \bar{S} \cdot \bar{S}^{-1}=\left(\mathcal{F} \cdot \overline{\mathcal{F}}^{-1}\right)_{+} & \left(\mathcal{F} \cdot \overline{\mathcal{F}}^{-1}\right)_{ \pm} \in G_{ \pm} .
\end{array}
$$

So that

Proposition 9. Given a dependence on an additional parameter 6 according to (72) and (175), introduce $H:=$ $F-\bar{F}$ and $\mathcal{H}:=\mathcal{F} \cdot \overline{\mathcal{F}}^{-1}$ where $F$ and $\bar{F}$ are defined (76), then

1. The dressing operators $W$ and $\bar{W}$ satisfy the following equations

$$
\partial_{6} W=-H_{-} \cdot W, \quad \partial_{6} \bar{W}=H_{+} \cdot \bar{W}, \quad \text { or } \quad T_{6} W=\mathcal{H}_{-} \cdot W, \quad T_{6} \bar{W}=\mathcal{H}_{+} \cdot \bar{W} .
$$

2. The Lax and Orlov-Schulman operators are subject to

$$
\begin{aligned}
& \partial_{6} L=\left[-H_{-}, L\right], \quad \partial_{6} M=\left[-H_{-}, M\right], \quad \partial_{6} C_{k k}=-\left[H_{-}, C_{k k}\right], \\
& \partial_{6} \bar{L}=\left[H_{+}, \bar{L}\right], \quad \partial_{6} \bar{M}=\left[H_{+}, \bar{M}\right], \quad \partial_{6} \bar{C}_{k k}=\left[H_{+}, \bar{C}_{k k}\right], \\
& \text { or } \\
& T_{6} L=\mathcal{H}_{-} \cdot L \cdot \mathcal{H}_{-}^{-1}, \quad T_{6} M=\mathcal{H}_{-} \cdot M \cdot \mathcal{H}_{-}^{-1}, \quad T_{6} C_{k k}=\mathcal{H}_{-} \cdot C_{k k} \cdot \mathcal{H}_{-}^{-1}, \\
& T_{6} \bar{L}=\mathcal{H}_{+} \cdot \bar{L} \cdot \mathcal{H}_{+}^{-1}, \quad T_{6} \bar{M}=\mathcal{H}_{+} \cdot \bar{M} \cdot \mathcal{H}_{+}^{-1}, \quad T_{6} \bar{C}_{k k}=\mathcal{H}_{+} \cdot \bar{C}_{k k} \cdot \mathcal{H}_{+}^{-1} .
\end{aligned}
$$




\subsubsection{String equations}

The factorization problem (9) depends decisively on the 'initial data' $g$. Now, we are going to see some consequences of the form of $g$ and derive the so called string equations. Let us suppose, that given two operators

$$
F_{0}:=\sum_{l, l^{\prime}=1}^{N} F_{l l^{\prime}}(n, \Lambda) E_{l l^{\prime}}, \quad \bar{F}_{0}=\sum_{l, l^{\prime}=1}^{N} \bar{F}_{l l^{\prime}}(n, \Lambda) E_{l l^{\prime}},
$$

we have the following constraint satisfied by $g$

$$
g \bar{F}_{0}=F_{0} g .
$$

Then, if

$$
F(M, L):=\sum_{l, l^{\prime}=1}^{N} F_{l l^{\prime}}(M, L) C_{l l^{\prime}}, \quad \quad \bar{F}(\bar{M}, \bar{L})=\sum_{l, l^{\prime}=1}^{N} \bar{F}_{l l^{\prime}}(\bar{M}, \bar{L}) \bar{C}_{l l^{\prime}}
$$

we have

$$
F(M, L)=\bar{F}(\bar{M}, \bar{L}) .
$$

We refer to these type of equations as string equations, see for example [25], and we have seen that they reflect properties like (78) of the initial condition $g$ in (91). Notice that the reduction of (41) is a particular case

of (78) with $\bar{F}_{0}:=\sum_{k=1}^{N} E_{k k} \Lambda^{-\ell_{\bar{k}}}$ and $F_{0}:=\sum_{k=1}^{N} E_{k k} \Lambda^{\ell_{k}}$, in which the Orlov-Schulman operator does not appear. This suggests an important family of diagonal string equations with

$$
F_{0}:=\sum_{k=1}^{N} E_{k k} F_{0, k}(n, \Lambda), \quad \bar{F}_{0}:=\sum_{k=1}^{N} E_{k k} \bar{F}_{0, k}(n, \Lambda) .
$$

The equations (56) are also a set of string equations; moreover, the invariance conditions under the additional flow (77) implies that $H=0$ or $\mathcal{H}=$ id so that we are lead to the string type equations of the form (79), namely

$$
F(M, L)=\bar{F}(\bar{M}, \bar{L}) \quad \text { or } \quad \mathcal{F}(M, L)=\overline{\mathcal{F}}(\bar{M}, \bar{L}) .
$$

This also follows from

$$
\begin{aligned}
& \partial_{6} g=\left(\partial_{6} h \cdot h^{-1}\right) g-g\left(\partial_{6} \bar{h} \cdot \bar{h}^{-1}\right)=F_{0} g-g \bar{F}_{0}, \\
& T_{6} g=\left(T_{6} h \cdot h^{-1}\right) \cdot g \cdot\left(T_{6} \bar{h} \cdot \bar{h}^{-1}\right)^{-1}=\mathcal{F}_{0} \cdot g \cdot \overline{\mathcal{F}}_{0}^{-1} .
\end{aligned}
$$

Observe if we consider arbitrary forms of $F_{0}, \bar{F}_{0}$ or $\mathcal{F}_{0}, \overline{\mathcal{F}}_{0}$ it will be same to deal with the description given here or the one obtained just setting $\bar{h}=\mathrm{id}$. However the situation is different if we consider the function $F_{0}$, $\bar{F}_{0}$ or $\mathcal{F}_{0}, \overline{\mathcal{F}}_{0}$ of diagonal type (80). In this case, to set $\bar{h}=$ id will generically imply to abandon the diagonal family for $F_{0}$.

\section{Appendix A: Congruences}

We will show here how to derive from the multi-component Toda hierarchy equations involving only the fields at each site $n \in \mathbb{Z}$; i.e. not mixing fields at different values of $n$, the sequence variable. This is particulary useful to show the role of the discrete multicomponent KP hierarchy in the multicomponent Toda hierarchy, which appears when we froze the bared continuous and discrete times.

Firstly, we present a queue observation

Proposition 10. Let us suppose that we have operators $R, \bar{R} \in \mathfrak{g}$ such that

$$
R W_{0}^{-1} \in \mathfrak{g}_{-}, \quad \bar{R} \bar{W}_{0}^{-1} \in \mathfrak{g}_{+}
$$

satisfying $R \cdot g=\bar{R}$. Then $R=\bar{R}=0$. 
Proof. We have

$$
\bar{R}=R g=R W^{-1} W g=R W^{-1} \bar{W} \Rightarrow \bar{R} \bar{W}^{-1}=R W^{-1}
$$

therefore $\bar{R} \bar{W}_{0}^{-1} \bar{S}^{-1}=R W_{0}^{-1} S^{-1}$ and recalling (82), and the fact that $S \in G_{-}$and $\bar{S} \in G_{+}$we conclude the statement.

Next, and without proof (which consist in a systematic and sometimes elaborated application of the previous result) we show the appearance of some well known integrable hierarchies within the multicomponent Toda hierarchy. We firstly point out that continuous variables, and for each value of $n$, we have solutions of the $N$-wave hierarchy and its modifications, moreover some discretizations of the modified $N$-wave equations are proposed. These results are just a manifestation of the fact that if we froze the bared times we are just dealing with a discrete $N$-component KP hierarchy in the spirit of [1. Next, we recover within this context the the quadrilateral lattice equations. Finally, we present what we call the dispersive Whitham hierarchy in complete analogy to the one proposed in [27]-28].

$N$-resonant wave equations and its modification We introduce

$$
\partial:=\partial_{11}+\cdots+\partial_{1 N}, \quad \bar{\partial}:=\partial_{1 \overline{1}}+\cdots+\partial_{1 \bar{N}},
$$

in terms of which we have

Theorem 4. The dressing operators satisfy the following equations

$$
\partial_{j a} W=Q_{j a}(W), \quad \partial_{j a} \bar{W}=Q_{j a}(\bar{W}),
$$

where

$$
Q_{j k}=u_{j k, j} \partial^{j}+u_{j k, j-1} \partial^{j-1}+\cdots+u_{j k, 0}, \quad Q_{j \bar{k}}=v_{j k, j} \bar{\partial}^{j}+v_{j k, j-1} \bar{\partial}^{j-1}+\cdots+v_{j k, 1} \bar{\partial}
$$

with the coefficients $u_{j k, i}, v_{j k, i}$ depending on $\partial_{1}^{s} \varphi_{r}, \bar{\partial}_{1}^{s} \bar{\varphi}_{r}$, respectively

$$
\begin{aligned}
u_{j k, j-i} & = \begin{cases}E_{k k}, & i=0, \\
\varphi_{i} E_{k k}-\sum_{a=0}^{i-1} u_{j k, j-a} \sigma_{j-a, i-a}, & i=1, \ldots, j,\end{cases} \\
v_{j k, j-i} & = \begin{cases}\bar{\varphi}_{0} E_{k k} \bar{\sigma}_{j, 0}^{-1}, & i=0, \\
\left(\bar{\varphi}_{i}-\sum_{a=0}^{i-1} \bar{v}_{j k, j-a} \bar{\sigma}_{j-a, i-a}\right) E_{k k} \bar{\sigma}_{j-i, 0}^{-1}, & i=1, \ldots, j-1,\end{cases}
\end{aligned}
$$

and

$$
\begin{aligned}
\sigma_{j, i} & :=\sum_{r=0}^{i-1}\left(\begin{array}{l}
j \\
r
\end{array}\right)\left(\partial^{r} \varphi_{i-r}\right), \\
\bar{\sigma}_{j, i} & :=\sum_{r=0}^{i}\left(\begin{array}{l}
j \\
r
\end{array}\right)\left(\bar{\partial}^{r} \bar{\varphi}_{i-r}\right) .
\end{aligned}
$$

Observe that $\sigma_{j 1}=\beta$ and $\bar{\sigma}_{j, 0}=\mathrm{e}^{\phi}$, and also that the first of the differential operators $Q_{j k}$ and $\bar{Q}_{j k}$ are given by

$$
\begin{aligned}
& Q_{j k}=E_{k k} \partial^{j}+\left[\beta, E_{k k}\right] \partial^{j-1}+\left(\left[\varphi_{2}, E_{k k}\right]-j E_{k k} \partial \beta-\left[\beta, E_{k k} \beta\right]\right) \partial^{j-2}+\cdots+u_{j k, 0}, \\
& Q_{j \bar{k}}=\mathrm{e}^{\phi} E_{k k} \mathrm{e}^{-\phi} \bar{\partial}^{j}+\left(\bar{\varphi}_{1} E_{k k}-\mathrm{e}^{\phi} E_{k k} \mathrm{e}^{-\phi}\left(\bar{\varphi}_{1}+j \bar{\partial} \mathrm{e}^{\phi}\right)\right) \mathrm{e}^{-\phi} \bar{\partial}^{j-1}+\cdots+v_{j k, 1} \bar{\partial} .
\end{aligned}
$$

Lemma 2. The only differential operators $Q=u_{j} \partial^{j}+\cdots+u_{0}$ and $\bar{Q}=v_{j} \bar{\partial}^{j}+\cdots+v_{0}$ such that

$$
Q(W)=0, \quad \bar{Q}(\bar{W})=0
$$

are

$$
Q=\bar{Q}=0 .
$$


Proof. - Let us suppose that $\sum_{i=0}^{j} u_{i} \partial^{i} W=0$ but $\left.\sum_{i=0}^{j} u_{i} \partial^{i} W=\left(\sum_{i=0}^{j}\left(u_{i}+\sum_{r=1}^{j-i} u_{i+r} \sigma_{i+r, r}\right) \Lambda^{i}+\mathfrak{g}_{-}\right)\right) W_{0}$. Thus, $u_{j}=u_{j-1}=\cdots=u_{0}=0$.

- Assume now that $\sum_{i=1}^{j} v_{i} \bar{\partial}^{i} \bar{W}=0$ and take into account that

$$
\sum_{i=1}^{j} v_{i} \bar{\partial}^{i} \bar{W}=\left(\sum_{i=1}^{j}\left(\sum_{r=0}^{j-i} v_{i+r} \bar{\sigma}_{i+r, r}\right) \Lambda^{-i}+\mathfrak{g}_{+}\right) \bar{W}_{0} .
$$

Thus, $v_{j}=v_{j-1}=\cdots=v_{1}=0$.

From this lemma it follows that

Proposition 11. The Zakharov-Shabat conditions

$$
\partial_{j k} Q_{i l}-\partial_{i l} Q_{j k}+\left[Q_{i l}, Q_{j k}\right]=0, \quad \partial_{j \bar{k}} Q_{i \bar{l}}-\partial_{i \bar{l}} Q_{j \bar{k}}+\left[Q_{i \bar{l}}, Q_{j \bar{k}}\right]=0
$$

hold.

Proof. Just consider the compatibility conditions of (83) together with Lemma 2

The site independent relations described in Theorem 4 constitute the $N$-wave hierarchy, for the non bared flows, and its modification for the bared flows. These multicomponent equations contains may integrable systems [17, for $N=1$ we have the KP equation in nonbared variables and the modified KP equation in bared variables, for $N=2$, the Davey-Stewartson equation in the $t$-variables and the Ishimori equation in the $\bar{t}$-variables. For $N=3$ we find the 3 -resonant wave system ( $t$-variables) and a modified version of it ( $\bar{t}$-variables).

Proposition 12. The $N$-wave equations

$$
\partial_{1 k}\left[\beta, E_{l l}\right]-\partial_{1 l}\left[\beta, E_{k k}\right]+\left[\left[\beta, E_{l l}\right],\left[\beta, E_{k k}\right]\right]=0 .
$$

and the modified $N$-wave equations

$$
\bar{\partial}_{1 k}\left(v_{l}\right)-\bar{\partial}_{1 l}\left(v_{k}\right)+v_{l} \bar{\partial}\left(v_{k}\right)-v_{k} \bar{\partial}\left(v_{l}\right)=0 .
$$

with $v_{k}:=\bar{\varphi}_{0} E_{k k} \bar{\varphi}_{0}^{-1}$ are satisfied.

Proof. The $N$-wave equations appear as the compatibility of the $Q_{1 k}=E_{k k} \partial_{1}+\left[\beta, E_{k k}\right]$. A "modified" $N$-wave system [17] appears for when one considers the compatibility $\bar{Q}_{1 k}=v_{k} \bar{\partial}, k=1, \ldots, N$.

Discrete versions of the modified $N$-wave equations For a fixed $l=1, \ldots, N$ let us introduce the following shift operator

$$
\bar{T}:=\sum_{\substack{\bar{k}=\overline{1}, \ldots, \bar{N} \\ \bar{k} \neq \bar{l}}} T_{(\bar{k}, \bar{l})},
$$

and the operator

$$
\bar{X}(A):=\sum_{k \neq l} T_{(\bar{k}, \bar{l})}(A) E_{k k}, \quad \quad P_{l}:=\sum_{k \neq l} E_{k k}, \quad A \in \mathfrak{g} .
$$

Finally we also introduce,

$$
\Omega_{k}:=V E_{k k} V^{-1}, \quad V:=E_{l l}+\bar{X}\left(\mathrm{e}^{\phi}\right),
$$

and the difference operators

$$
\Delta_{(\bar{k}, \bar{l})}:=T_{(\bar{k}, \bar{l})}-1, \quad \bar{\Delta}:=\sum_{k \neq l} \Delta_{(\bar{k}, \bar{l})}=\bar{T}-(N-1) .
$$

Proposition 13. The dressing operators $W$ and $\bar{W}$ satisfy

$$
\Delta_{(\bar{k}, \bar{l})}(W)=\Omega_{k} \bar{\Delta}(W), \quad \Delta_{(\bar{k}, \bar{l})}(\bar{W})=\Omega_{k} \bar{\Delta}(\bar{W}) .
$$


Conjugate nets and quadrilateral lattices We show now the role of conjugate nets and quadrilateral lattices as a part of the multicomponent Toda hierarchy. For this aim we first prove

Proposition 14. If $\epsilon_{i} \in M_{N}(\mathbb{C}), i=1,2$ are such that $\epsilon_{1} E_{k k}=\epsilon_{2} E_{l l}$ then

$$
\begin{aligned}
\epsilon_{1}\left(\partial_{1 k}-\beta E_{k k}\right) W & =\epsilon_{2}\left(T_{K}-\left(T_{K} \beta E_{l l}+\square_{N}-E_{l l}-\pi_{a}\right)\right) W, & & K=(l, a) \\
\epsilon_{1}\left(\partial_{1 k}-\beta E_{k k}\right) \bar{W} & =\epsilon_{2}\left(T_{K}-\left(T_{K} \beta E_{l l}+\square_{N}-E_{l l}-\pi_{a}\right) \bar{W},\right. & & K=(l, a) \\
\epsilon_{1} \mathrm{e}^{-\phi} \partial_{1 \bar{k}} W & =\epsilon_{2} \mathrm{e}^{-T_{K} \phi}\left(\Delta_{K}+\pi_{a}\right) W, & & K=(\bar{l}, a) \\
\epsilon_{1} \mathrm{e}^{-\phi} \partial_{1 \bar{k}} \bar{W} & =\epsilon_{2} \mathrm{e}^{-T_{K} \phi}\left(\Delta_{K}+\pi_{a}\right) \bar{W}, & & K=(\bar{l}, a) .
\end{aligned}
$$

A particular consequence is

$$
\begin{aligned}
\epsilon_{1}\left(\varphi_{2} E_{k k}+\partial_{1 k} \varphi_{1}-\varphi_{1} E_{k k} \varphi_{1}\right)=\epsilon_{2}\left(T_{(k, l)} \varphi_{2} E_{k k}+T_{k l} \varphi_{1}\left(\rrbracket_{N}-E_{l l}\right.\right. & \left.-E_{k k}\right) \\
& \left.+E_{l l}-\left(T_{k l} \varphi_{1} E_{k k}+\rrbracket_{N}-E_{l l}-E_{k k}\right) \varphi_{1}\right) .
\end{aligned}
$$

If we right multiply this relation by $E_{m^{\prime} m^{\prime}}$ with $m^{\prime} \neq k$ and we take

1. $\epsilon_{1}=E_{m m}$ with $m \neq k$ and $\epsilon_{2}=0$

2. $\epsilon_{1}=0$ and $\epsilon_{2}=E_{m m}$ with $m \neq k, l$

3. $\epsilon_{1}=0$ and $\epsilon_{2}=E_{l l}$

4. $\epsilon_{1}=\epsilon_{2}=E_{k k}$

we find

$$
\begin{aligned}
\partial_{1 k} \beta_{m m^{\prime}}-\beta_{m k} \beta_{k m^{\prime}} & =0, & & \text { for } m, m^{\prime} \neq k \\
\Delta_{(k, l)} \beta_{m m^{\prime}}-\left(T_{(k, l)} \beta_{m k}\right) \beta_{k m^{\prime}} & =0, & & \text { for } m, m^{\prime} \neq k, l, \\
\left(T_{(k, l)} \beta_{m k}\right) \beta_{k l}+\beta_{m l} & =0, & & m \neq k, l, \\
T_{(k, l)} \beta_{l m^{\prime}}-\left(T_{(k, l)} \beta_{l k}\right) \beta_{k m^{\prime}} & =0, & & m^{\prime} \neq k, l, \\
\left(T_{(k, l)} \beta_{l k}\right) \beta_{k l}-1 & =0, & & \\
\partial_{1 k} \log \beta_{k m^{\prime}}-\frac{T_{(k, l)} \beta_{k m^{\prime}}}{\beta_{k m^{\prime}}}+\Delta_{(k, l)} \beta_{k k} & =0, & & m^{\prime} \neq l, \\
\partial_{1 k} \log \beta_{k l}+\Delta_{(k, l)} \beta_{k k} & =0 . & &
\end{aligned}
$$

The dispersionfull Toda-Whitham hierarchy We fix $l \in \mathbb{S}$ and consider the shifts $T_{(a, l)}$ for $a \in \mathcal{S}$ with $a \neq l$, and as we can not put $a=l$ for $a^{\prime} \in \mathcal{S}, a^{\prime} \neq l$,

Proposition 15. 1. For $a^{\prime}, l, a^{\prime} \neq l$, there exists scalar operators

$$
\begin{aligned}
\mathscr{B}_{j l} & =T_{\left(l, a^{\prime}\right)}^{j}+B_{j l, j-1} T_{\left(l, a^{\prime}\right)}^{-j+1}+\cdots+B_{j l, 0}, \\
\alpha_{j l} & =\partial_{1 l}^{j}+\alpha_{j l, j-2} \partial_{1 l}^{j-2}+\cdots+\alpha_{j l, 0}
\end{aligned}
$$

where the coefficients $B_{j l, i}$ and $\alpha_{j l, i}$ are scalar polynomials in the $T_{\left(l, a^{\prime}\right)}$-shifts or the $\partial_{1 l}$-derivatives of $\beta_{l l}, \varphi_{2, l l} \ldots, \varphi_{j, l l}$, respectively, for example $B_{j l, j-1}=\beta_{l l}-T_{\left(l, a^{\prime}\right)}^{j} \beta_{l l}$ such that

$$
\partial_{j l}\left(E_{l l} W\right)=\mathscr{B}_{j l}\left(E_{l l} W\right)=\alpha_{j l}\left(E_{l l} W\right), \quad \partial_{j l}\left(E_{l l} \bar{W}\right)=\mathscr{B}_{j l}\left(E_{l l} \bar{W}\right)=\alpha_{j l}\left(E_{l l} \bar{W}\right),
$$

2. For $a \neq l$ there exists scalar operators

$$
\mathscr{B}_{j a}=B_{j a, j} T_{(a, l)}^{j}+\cdots+B_{j a, 1} T_{(a, l)}
$$

where $B_{j a, i}$ are scalar polynomials in the $T_{(l, a)}$-shifts of $\beta_{l k}, \varphi_{2, l k}, \ldots, \varphi_{j, l k}$ when $a=k$ and of $\bar{\varphi}_{0, l k}, \ldots, \bar{\varphi}_{j-1, l k}$ for $a=\bar{k}$, for example

$$
B_{j a, j}= \begin{cases}\frac{\beta_{l k}}{T_{(k, l)}^{j}\left(\beta_{l k}\right)}, & a=k \in \mathbb{S}, \\ \frac{\bar{\varphi}_{0, l k}}{T_{(\bar{k}, l)}^{j}\left(\bar{\varphi}_{0, l k}\right)}, & a=\bar{k} \in \overline{\mathbb{S}},\end{cases}
$$

such that for $a \neq l$

$$
\partial_{j a}\left(E_{l l} W\right)=\mathscr{B}_{j a}\left(E_{l l} W\right) \quad \partial_{j a}\left(E_{l l} \bar{W}\right)=\mathscr{B}_{j a}\left(E_{l l} \bar{W}\right) .
$$




\section{Appendix B: Proofs of Propositions}

- Proposition 2 Obviously (23) is implied by (20)-(22). It is also easy to conclude that (21) and (22) follow from (23). The non trivial part of the proposition is to prove that (23) implies (20):

$$
\begin{aligned}
T_{(a, b)} T_{(c, d)} & =T_{(a, c)} T_{(c, b)} T_{(c, b)} T_{(b, d)}=T_{(a, c)} T_{(c, b)} T_{(b, d)} T_{(c, b)} \\
& =T_{(a, c)} T_{(c, d)} T_{(c, b)}=T_{(c, d)} T_{(a, c)} T_{(c, b)}=T_{(c, d)} T_{(a, b)} .
\end{aligned}
$$

- Proposition 3 We only need to show that (37) implies (33) as the reverse is evident. We proceed as in the proof of Proposition 2

$$
\begin{aligned}
\left(T_{(a, b)} \omega_{(c, d)}\right) \omega_{(a, b)} & \left.=\left(T_{(a, b)}\left(T_{(b, d)} \omega_{(c, b)}\right) \omega_{(b, d)}\right)\right)\left(T_{(a, c)} \omega_{(c, b)}\right) \omega_{(a, c)} \\
& =\left(T_{(a, b)}\left(T_{(b, d)} \omega_{(c, b)}\right)\right)\left(T_{(a, c)}\left(T_{(c, b)} \omega_{(b, d)}\right) \omega_{(c, b)}\right) \omega_{(a, c)} \\
& =\left(T_{(a, b)}\left(T_{(b, d)} \omega_{(c, b)}\right)\right)\left(T_{(a, c)} \omega_{(c, d)}\right) \omega_{(a, c)} \\
& =\left(T_{(c, d)}\left(T_{(a, c)} \omega_{(c, b)}\right)\right)\left(T_{(c, d)} \omega_{(a, c)}\right) \omega_{(c, d)} \\
& =\left(T_{(c, d)}\left(T_{(a, c)} \omega_{(c, b)}\right) \omega_{(a, c)}\right) \omega_{(c, d)} \\
& =\left(T_{(c, d)} \omega_{(a, b)}\right) \omega_{(c, d)} .
\end{aligned}
$$

- Proposition 4 We do not prove the differential case and refer the reader to [30]. Therefore we proceed to the remaining cases involving discrete times.

1. We start by proving (32). First, from the definition (19) we deduce that

$$
\begin{aligned}
\partial_{j a} \omega_{K} \cdot \omega_{K}^{-1} & =\partial_{j a}\left(\mathcal{U}_{K} \cdot \overline{\mathcal{U}}_{K}^{-1}\right)_{-} \cdot\left(\mathcal{U}_{K} \cdot \overline{\mathcal{U}}_{K}^{-1}\right)_{-}^{-1}+\left(\mathcal{U}_{K} \cdot \overline{\mathcal{U}}_{K}^{-1}\right)_{-} \partial_{j a} \mathcal{U}_{K} \cdot \mathcal{U}_{K}^{-1}\left(\mathcal{U}_{K} \cdot \overline{\mathcal{U}}_{K}^{-1}\right)_{-}^{-1} \\
& =\partial_{j a}\left(\mathcal{U}_{K} \cdot \overline{\mathcal{U}}_{K}^{-1}\right)_{-} \cdot\left(\mathcal{U}_{K} \cdot \overline{\mathcal{U}}_{K}^{-1}\right)_{-}^{-1}+\left(\mathcal{U}_{K} \cdot \overline{\mathcal{U}}_{K}^{-1}\right)_{-}\left(B_{j a}-\mathcal{U}_{K} B_{j a} \mathcal{U}_{K}^{-1}\right)\left(\mathcal{U}_{K} \cdot \overline{\mathcal{U}}_{K}^{-1}\right)_{-}^{-1} \\
& =\partial_{j a}\left(\mathcal{U}_{K} \cdot \overline{\mathcal{U}}_{K}^{-1}\right)_{-} \cdot\left(\mathcal{U}_{K} \cdot \overline{\mathcal{U}}_{K}^{-1}\right)_{-}^{-1}+\left(\mathcal{U}_{K} \cdot \overline{\mathcal{U}}_{K}^{-1}\right)_{-} B_{j a}\left(\mathcal{U}_{K} \cdot \overline{\mathcal{U}}_{K}^{-1}\right)_{-}^{-1}-\omega_{K} B_{j a} \omega_{K}^{-1} \\
& \text { and similarly } \\
& =\partial_{j a}\left(\mathcal{U}_{K} \cdot \overline{\mathcal{U}}_{K}^{-1}\right)_{+} \cdot\left(\mathcal{U}_{K} \cdot \overline{\mathcal{U}}_{K}^{-1}\right)_{+}^{-1}+\left(\mathcal{U}_{K} \cdot \overline{\mathcal{U}}_{K}^{-1}\right)_{+} B_{j a}\left(\mathcal{U}_{K} \cdot \overline{\mathcal{U}}_{K}^{-1}\right)_{+}^{-1}-\omega_{K} B_{j a} \omega_{K}^{-1}
\end{aligned}
$$

so that

$$
\begin{aligned}
\partial_{j a} \omega_{K} \cdot \omega_{K}^{-1}+\omega_{K} B_{j a} \omega_{K}^{-1} & =\partial_{j a}\left(\mathcal{U}_{K} \cdot \overline{\mathcal{U}}_{K}^{-1}\right)_{-} \cdot\left(\mathcal{U}_{K} \cdot \overline{\mathcal{U}}_{K}^{-1}\right)_{-}^{-1}+\left(\mathcal{U}_{K} \cdot \overline{\mathcal{U}}_{K}^{-1}\right)_{-} B_{j a}\left(\mathcal{U}_{K} \cdot \overline{\mathcal{U}}_{K}^{-1}\right)_{-}^{-1} \\
& =\partial_{j a}\left(\mathcal{U}_{K} \cdot \overline{\mathcal{U}}_{K}^{-1}\right)_{+} \cdot\left(\mathcal{U}_{K} \cdot \overline{\mathcal{U}}_{K}^{-1}\right)_{+}^{-1}+\left(\mathcal{U}_{K} \cdot \overline{\mathcal{U}}_{K}^{-1}\right)_{+} B_{j a}\left(\mathcal{U}_{K} \cdot \overline{\mathcal{U}}_{K}^{-1}\right)_{+}^{-1}
\end{aligned}
$$

Now, using (33) and the commuting character of the Lax operators we get

$$
\begin{aligned}
T_{K} B_{j a} & =\left(\mathcal{U}_{K} \cdot \overline{\mathcal{U}}_{K}^{-1}\right)_{-} \mathcal{R}_{j a}\left(\mathcal{U}_{K} \cdot \overline{\mathcal{U}}_{K}^{-1}\right)_{-}^{-1}-T_{K}\left(\mathcal{R}_{j a}-\overline{\mathcal{R}}_{j a}\right)_{-} \\
& =\left(\mathcal{U}_{K} \cdot \overline{\mathcal{U}}_{K}^{-1}\right)_{+} \overline{\mathcal{R}}_{j a}\left(\mathcal{U}_{K} \cdot \overline{\mathcal{U}}_{K}^{-1}\right)_{+}^{-1}+T_{K}\left(\mathcal{R}_{j a}-\overline{\mathcal{R}}_{j a}\right)_{-}
\end{aligned}
$$

and we deduce for $I:=\partial_{j a} \omega_{K} \cdot \omega_{K}^{-1}+\omega_{K} B_{j a} \omega_{K}^{-1}-T_{K} B_{j a}$ the following expressions

$$
\begin{aligned}
I & =\partial_{j a}\left(\mathcal{U}_{K} \cdot \overline{\mathcal{U}}_{K}^{-1}\right)_{-} \cdot\left(\mathcal{U}_{K} \cdot \overline{\mathcal{U}}_{K}^{-1}\right)_{-}^{-1}-\left(\mathcal{U}_{K} \cdot \overline{\mathcal{U}}_{K}^{-1}\right)_{-}\left(\mathcal{R}_{j a}-\overline{\mathcal{R}}_{j a}\right)_{-}\left(\mathcal{U}_{K} \cdot \overline{\mathcal{U}}_{K}^{-1}\right)_{-}^{-1}+T_{K}\left(\mathcal{R}_{j a}-\overline{\mathcal{R}}_{j a}\right)_{-} \\
& =\partial_{j a}\left(\mathcal{U}_{K} \cdot \overline{\mathcal{U}}_{K}^{-1}\right)_{+} \cdot\left(\mathcal{U}_{K} \cdot \overline{\mathcal{U}}_{K}^{-1}\right)_{+}^{-1}+\left(\mathcal{U}_{K} \cdot \overline{\mathcal{U}}_{K}^{-1}\right)_{+}\left(\mathcal{R}_{j a}-\overline{\mathcal{R}}_{j a}\right)_{+}\left(\mathcal{U}_{K} \cdot \overline{\mathcal{U}}_{K}^{-1}\right)_{+}^{-1}-T_{K}\left(\mathcal{R}_{j a}-\overline{\mathcal{R}}_{j a}\right)_{+} \cdot
\end{aligned}
$$

which hold only if $I=0$, as desired.

2. Let us now prove (33). From (19) and (30) we get

$$
T_{K} \omega_{K^{\prime}}=T_{K}\left(\mathcal{U}_{K^{\prime}} \overline{\mathcal{U}}_{K^{\prime}}^{-1}\right)_{-} \cdot \omega_{K} \mathcal{U}_{K^{\prime}} \omega_{K}^{-1}=T_{K}\left(\mathcal{U}_{K^{\prime}} \overline{\mathcal{U}}_{K^{\prime}}^{-1}\right)_{+} \cdot \omega_{K} \overline{\mathcal{U}}_{K^{\prime}} \omega_{K}^{-1}
$$

or using (19) again

$$
T_{K} \omega_{K^{\prime}} \cdot \omega_{K}=T_{K}\left(\mathcal{U}_{K^{\prime}} \overline{\mathcal{U}}_{K^{\prime}}^{-1}\right)_{-} \cdot\left(\mathcal{U}_{K} \overline{\mathcal{U}}_{K}^{-1}\right)_{-} \mathcal{U}_{K} \mathcal{U}_{K^{\prime}}=T_{K}\left(\mathcal{U}_{K^{\prime}} \overline{\mathcal{U}}_{K^{\prime}}^{-1}\right)_{+} \cdot\left(\mathcal{U}_{K} \overline{\mathcal{U}}_{K}^{-1}\right)_{+} \overline{\mathcal{U}}_{K} \overline{\mathcal{U}}_{K^{\prime}}
$$

Then, we deduce

$$
\left(T_{K} \omega_{K^{\prime}} \cdot \omega_{K}\right)_{+}=\left(\mathcal{U}_{K} \mathcal{U}_{K^{\prime}}\right)_{+}, \quad\left(T_{K} \omega_{K^{\prime}} \cdot \omega_{K}\right)_{-}=\left(\overline{\mathcal{U}}_{K^{\prime}}^{-1} \overline{\mathcal{U}}_{K}^{-1}\right)_{-}
$$

Interchanging $K \leftrightarrow K^{\prime}$ and recalling the commuting character of the Lax operators we get the desired result. 
- Proposition [ Let $a_{i j}$ denote the elements of the bi-infinite matrix $g_{k_{1} k_{2}}$, we now proceed to analyze the meaning of (48) in different situations:

- Block Hankel case Let us assume that $\ell_{k_{1}} \ell_{\bar{k}_{2}}>0$. In particular let us discuss the case where both integers are positive. If we start from the element $a_{i j}$ the equation (48) says that it is equal to some other element. To determine the this element in the matrix we observe that (48) requires to move in the $i$-th row $\ell_{k_{1}}+\ell_{\bar{k}_{2}}$ positions to the right and in the diagonal passing through that position go left $\ell_{k_{1}}$ positions on this diagonal, i.e. go up $\ell_{k_{1}}$ positions and to the left also $\ell_{k_{1}}$ positions. This gives us the block structure over off-diagonals as illustrated below.

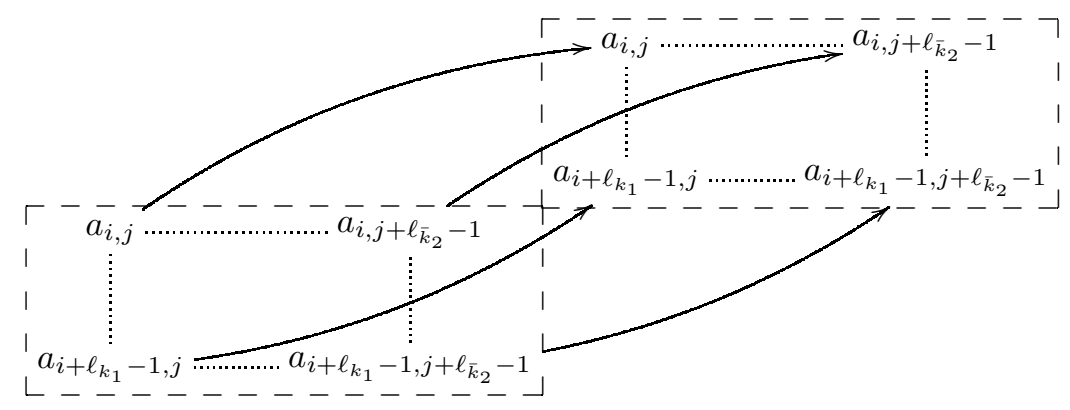

For negative integers $\ell_{k_{1}}, \ell_{\bar{k}_{2}}<0$ we have a similar discussion, replacing right motions in the row with left motions and up motions in the diagonal with down motions in the diagonal, and the same block Hankel structure appears.

- Block Toeplitz case We now assume that $\ell_{k_{1}} \ell_{\bar{k}_{2}}<0$. Suppose that $\ell_{k_{1}}$ is positive. Then, when $\ell_{k_{1}}>\left|\ell_{\bar{k}_{2}}\right|$ if we start from the element $a_{i j}$ the equation (48) says that it is equal to some other element, say $a_{i^{\prime} j^{\prime}}$. To determine the row $i^{\prime}$ and column $j^{\prime}$, we observe that (48) tell us to advance in the $i$-th row $\ell_{k_{1}}-\left|\ell_{\bar{k}_{2}}\right|$ positions to the right and in the diagonal passing through that position go left $\ell_{k_{1}}$ positions on this diagonal, i.e. go up $\ell_{k_{1}}$ positions and to the left also $\ell_{k_{1}}$ positions. So that we have

$$
a_{i j}=a_{i-\ell_{k_{1}}, j+\ell_{k_{1}}-\left|\ell_{\bar{k}_{2}}\right|-\ell_{k_{1}}}=a_{i-\ell_{k_{1}}, j-\left|\ell_{\bar{k}_{2}}\right|} \cdot
$$

For the case $\ell_{k_{1}}<\left|\ell_{\bar{k}_{2}}\right|$ we use $g_{j, k_{1} k_{2}}(n)=g_{j+\left|\ell_{\bar{k}_{2}}\right|-\ell_{k_{1}}, k_{1} k_{2}}\left(n+\ell_{k_{1}}\right)$ so that we move $\left|\ell_{\bar{k}_{2}}\right|-\ell_{k_{1}}$ positions to the right and on the diagonal $\ell_{k_{1}}$ positions down, which amounts to $\ell_{k_{1}}$ rows down and $\ell_{k_{1}}$ columns right, i.e.

$$
a_{i j}=a_{i-\ell_{k_{1}}, j+\left|\ell_{\bar{k}_{2}}\right|-\ell_{k_{1}}+\ell_{k_{1}}}=a_{i-\ell_{k_{1}}, j-\left|\ell_{\bar{k}_{2}}\right|}
$$

and we get the same result, which immediately tell us about the block structure over diagonals as illustrated below.

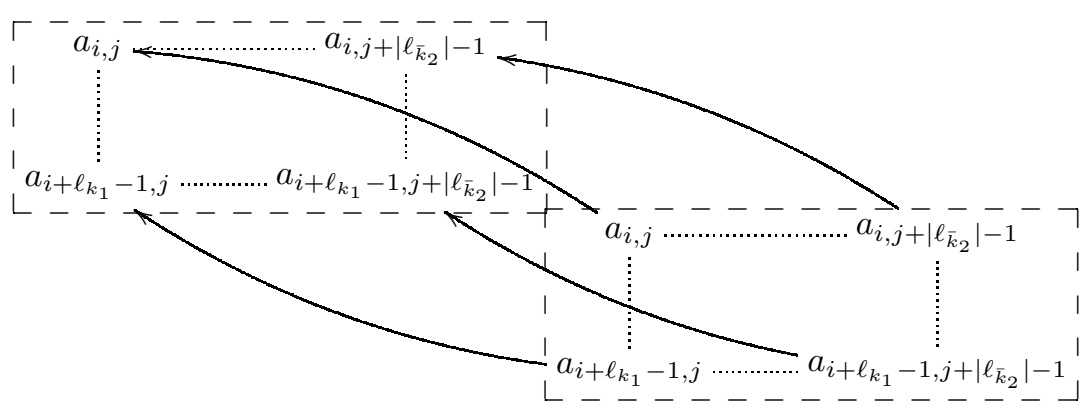

A similar discussion goes on for the case of negative $\ell_{k_{1}}$ and positive $\ell_{\bar{k}_{2}}$. 
- The case $\ell_{k_{1}}=0$ with $\ell_{\bar{k}_{2}} \neq 0$ gives $g_{j, k_{1} k_{2}}(n)=g_{j+\ell_{\bar{k}_{2}}, k_{1} k_{2}}(n)$, which implies diagonal band structure, whether for $\ell_{\bar{k}_{2}}=0$ with $\ell_{k_{1}} \neq 0$ gives $g_{j, k_{1} k_{2}}(n)=g_{j+\ell_{k_{1}}, k_{1} k_{2}}\left(n+\ell_{k_{1}}\right)$, which describes a $\ell_{k_{1}} \times \ell_{k_{1}}$ block structure. Notice that these two cases can only exist for two or more components.

- Proposition 7 Let us compute

$$
\begin{aligned}
& M=W n W^{-1}=S W_{0} n W_{0}^{-1} S^{-1}, \\
& \bar{M}=\bar{W} n \bar{W}^{-1}=\bar{S} \bar{W}_{0} n \bar{W}_{0}^{-1} \bar{S}^{-1},
\end{aligned}
$$

for this aim we must take into account that

$$
\begin{aligned}
& \mu:=W_{0} n W_{0}^{-1}=n+\nu, \quad \nu=\sum_{k=1}^{N} E_{k k}\left(s_{k}+\sum_{j=1}^{\infty} j t_{j k} \Lambda^{j}\right), \\
& \bar{\mu}:=\bar{W}_{0} n \bar{W}_{0}^{-1}=n+\bar{\nu}, \quad \bar{\nu}=-\sum_{k=1}^{N} E_{k k}\left(s_{\bar{k}}+\sum_{j=1}^{\infty} j t_{j \bar{k}} \Lambda^{-j}\right) .
\end{aligned}
$$

Therefore, from (87) and (88) we deduce that

$$
\begin{aligned}
& M=S \mu S^{-1}=S n S^{-1}+\sum_{k=1}^{N} C_{k k}\left(s_{k}+\sum_{j=1}^{\infty} j t_{j k} L^{j}\right), \\
& \bar{M}=\bar{S} \bar{\mu} \bar{S}^{-1}=\bar{S} n \bar{S}^{-1}-\sum_{k=1}^{N} \bar{C}_{k k}\left(s_{\bar{k}}+\sum_{j=1}^{\infty} j t_{j \bar{k}} \bar{L}^{-j}\right) .
\end{aligned}
$$

Finally,

$$
\begin{aligned}
\mathcal{M} & :=S n S^{-1}=\left(1+\beta(n) \Lambda^{-1}+\varphi_{2}(n) \Lambda^{-2}+\cdots\right) n\left(1+\beta(n) \Lambda^{-1}+\varphi_{2}(n) \Lambda^{-2}+\cdots\right)^{-1} \\
& =n-\beta(n) \Lambda^{-1}+\cdots \\
\overline{\mathcal{M}} & :=\bar{S} n \bar{S}^{-1}=\left(\mathrm{e}^{\phi(n)}+\bar{\varphi}_{1}(n) \Lambda+\cdots\right) n\left(\mathrm{e}^{\phi(n)}+\bar{\psi}_{1}(n) \Lambda+\cdots\right)^{-1} \\
& =n+\bar{\varphi}_{1}(n) \mathrm{e}^{-\phi(n+1)} \Lambda+\cdots .
\end{aligned}
$$

- Proposition 8 Let us take $W$ of Theorem 2 and consider $\Theta_{k l}:=W^{-1} C_{k l} W$ which satisfy $\left[\Theta_{k l}, \Lambda\right]=$ $\left[\Theta_{k l}, n\right]=0$ and hence $\Theta_{k l}$ do not depends on $\Lambda$ nor on $n$. Now,

$$
\begin{aligned}
& E_{k^{\prime} k^{\prime}} \Theta_{k l}=\delta_{k^{\prime} k} \Theta_{k^{\prime} l}, \quad \Theta_{k l} E_{k^{\prime} k^{\prime}}=\delta_{l k^{\prime}} \Theta_{k l} \quad \Rightarrow \quad \Theta_{k l}=\vartheta_{k l} E_{k l}, \quad \vartheta_{k l} \in \mathbb{C}, \\
& E_{k^{\prime} k^{\prime}} \bar{\Theta}_{k l}=\delta_{k^{\prime} k} \bar{\Theta}_{k^{\prime} l}, \quad \bar{\Theta}_{k l} E_{k^{\prime} k^{\prime}}=\delta_{l k^{\prime}} \bar{\Theta}_{k l} \quad \Rightarrow \quad \bar{\Theta}_{k l}=\bar{\vartheta}_{k l} E_{k l}, \quad \bar{\vartheta}_{k l} \in \mathbb{C} .
\end{aligned}
$$

Thus,

$$
\begin{array}{r}
C_{k l}=W \Theta_{k l} W^{-1}=S W_{0} \vartheta_{k l} E_{k l} W_{0}^{-1} S^{-1}=\vartheta_{k l} L^{s_{k}-s_{l}} \mathrm{e}^{\sum_{j \geqslant 1}\left(t_{j k}-t_{j l}\right) L^{j}}\left(E_{k l}+\mathfrak{g}_{-}\right) \\
\Rightarrow \vartheta_{k l}=1 \Rightarrow C_{k l}=W E_{k l} W^{-1} .
\end{array}
$$

\section{Acknowledgements}

The authors wish to thank the Spanish Ministerio de Ciencia e Innovación, research projects FIS2005-00319 and FIS2008-00200, and acknowledge the support received from the European Science Foundation (ESF) and the activity entitled Methods of Integrable Systems, Geometry, Applied Mathematics (MISGAM). This paper was finished during the research visits of one of the authors (MM) to the Universite Catholique de Louvain and to the Scuola Internazionale Superiore di Studi Avanzati/International School for Advanced Studies (SISSA) in Trieste, MM wish to thanks Prof. van Moerbeke and Prof. Dubrovin for their warm hospitality, acknowledge economical support from MISGAM and SISSA and reckons different conversations with P. van Moerbeke, T. Grava, G. Carlet and M. Caffasso. 


\section{References}

[1] M. Adler and P. van Moerbeke, Comm. Math. Phys. 203 (1999) 185 .

[2] M. Adler and P. van Moerbeke, Comm. Pure App. Math. 54 (2001) 153.

[3] M. Adler, P. van Moerbeke, and P. Vanhaecke, Comm. Math. Phys. 286 (2009) 1.

M.Adler. J. Delépine and P. van Moerbeke, Comm. Pure App. Math. 62 (2009) 334.

E. Daems and A.B.J. Kuijlaars, J. of Approx. Theory 146 (2007) 91.

[4] M. J. Bergvelt and A. P. E. ten Kroode, Pacific J. Math. 171 (1995) 23.

[5] A. Böttcher, M. Embree, and V. I. Sokolov, Lin. Alg. App. 343-344 (2002) 101.

T. Strohmer, Lin. Alg. App. 343-344 (2002) 321.

[6] M. Cafasso, Matrix biorthogonal polynomials on the unit circle and non-abelian Ablowitz-Ladik hierarchy. arXiv:0804.3572v2 [math.CA] 23 Apr 2008

[7] G. Carlet, J. Phys. A: Math. Gen. 39 (2006) 9411.

[8] E. Date, M. Jimbo, M. Kashiwara, and T. Miwa, J. Phys. Soc. Japan 40 (1981) 3806.

[9] A. Doliwa and P.M. Santini, Phys. Lett. A 233 (1997) 365.

M. Mañas, A. Doliwa and P.M. Santini, Phys. Lett. A 232 (1997) 99.

A. Doliwa, P.M. Santini and M. Mañas, J. Math. Phys. 41 (2000) 944.

[10] B. Eynard, An Introduction to Random Matrices, lectures given at Saclay, October 2000, http://www-spht.cea.fr/articles/t01/014/.

[11] P. di Francesco, P. Ginsparg and Z. Zinn-Justin, Phys. Rept. 254 (1995) 1.

[12] E. Getzler, The Toda conjecture, math.AG/0108108. Published in: Symplectic geometry and mirror symmetry (Seoul, 2000), 5179, World Sci. Publishing, River Edge, NJ, 2001.

G. Carlet, Theor. Math. Phys. 137 (2003) 1390.

G. Carlet, B. Dubrovin, and Y. Zhang, Moscow Math. J. 4 (2004) 313.

B. Dubrovin and Y. Zhang, Comm. Math. Phys. 250 (2004) 161.

[13] J. Harnad. and A. Yu. Orlov, Theor. Math. Phys, 152 (2007) 1099

[14] A. Gerasimov, A. Marshakov, A. Mironov, A. Morozov and A. Orlov, Nuc. Phys. B 357 (1991) 565.

[15] V. G. Kac and J. W. van de Leur, J. Math. Phys. 44 (2003) 3245.

[16] I. M. Krichever, Comm. Pure. Appl. Math. 47 (1994) 437.

[17] B. G. Konopelchenko and W. Oevel, "Matrix Sato Theory and Integrable Equations in 2+1 Dimensions" in Procceding NEEDS'91, Baia Verde, Italy, June 1991.

[18] E. J. Martinec, Comm. Math. Phys. 138 (1991) 437.

[19] M. Mañas, L. Martínez Alonso, and E. Medina, J. Phys. A: Math. Gen. 35 (2002) 401.

L. Martínez Alonso and M. Mañas, J. Math. Phys. 443294 (2003).

F. Guil, M. Mañas, and L. Martínez Alonso, J. Phys. A: Math. Gen. 36 (2003) 4047.

F. Guil, M. Mañas, and L. Martínez Alonso, J. Phys. A: Math. Gen. 36 (2003) 6457.

M. Mañas, J. Phys. A: Math. Gen. 37 (2004) 9195.

M. Mañas, J. Phys. A: Math. Gen. 37 (2004) 11191.

[20] M. Mañas, L. Martínez Alonso, and E. Medina, J. Phys.A: Math. Gen. 33 (2000) 2871.

M. Mañas, L. Martínez Alonso, and E. Medina, J. Phys.A: Math. Gen. 33 (2000) 7181.

[21] L. Martínez Alonso and E. Medina, J. Phys. A: Math. Gen. 40 (2007) 14223.

[22] L. Martínez Alonso and E. Medina, Multiple orthogonal polynomials, string equations and the large-n limit , arXiv:0812.3817.

[23] A. Yu Orlov and E. I. Schulman, Lett. Math. Phys. 12 (1986) 171.

[24] A. G. Reimann and M. A. Semenov-Tyan-Shanski, J. Math. Sci. 31 (1985) 3399.

[25] K. Takasaki, Commun. Math. Phys. 181 (1996) 131.

M. Mañas, E. Medina and L. Martínez Alonso, J. Phys.A: Math. Gen. 39 (2006) 2349.

L. Martínez Alonso, E. Medina and M. Mañas, J. Math. Phys. 47 (2006) 083512. 
[26] K. Takasaki and T. Takebe, Rev. Math. Phys. 7 (1995) 743.

[27] K. Takasaki, "Dispersionless integrable hierarchies revisited", talk delivered at SISSA at september 2005 (MISGAM program).

[28] K. Takasaki and T. Takebe, Physica D 235 (2007) 109.

[29] K. Takasaki and T. Takebe, Löwner equations, Hirota equations and reductions of universal Whitham hierachy arXiv:0808.1444

[30] K. Ueno and K. Takasaki, Adv. Stud. Pure Math. 4 (1984) 1.

[31] H. Widom, Trans. American Math. Soc. 121 (1966) 1.

M. Van Barel, V. Ptak, Z. Vaverín, Lin. Alg. App. 332-334 (2001) 583.

[32] P. B. Wiegmann and A. Zabrodin, Comm. Math. Phys. 213 (2000) 523.

M. Mineev-Weinstein, P.B. Wiegmann, and A. Zabrodin, Phys. Rev. Lett. 84 (2000) 5106

I. M. Krichever, M. Mineev-Weinstein, P. B. Wiegmann, and A. Zabrodin, Physica D198 (2004) 1.

I. M. Krichever, A. Marshakov and A. Zabrodin, Comm. Math. Phys. 259 (2005) 1. 